\title{
Article \\ Elevated Circulating Microparticle Subpopulations in Incidental Cerebral White Matter Hyperintensities: A Multimodal Study
}

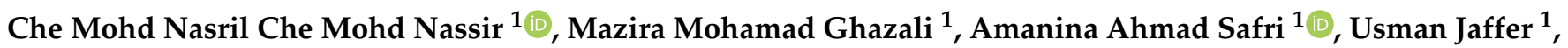 \\ Wan Zaidah Abdullah ${ }^{2,3}$, Nur Suhaila Idris ${ }^{3,4}$ and Mustapha Muzaimi 1,3,*(D) \\ 1 Department of Neurosciences, School of Medical Sciences, Universiti Sains Malaysia, Kubang Kerian 16150, \\ Kelantan, Malaysia; nasrilche123@gmail.com (C.M.N.C.M.N.); \\ mazira.mohamadghazali@gmail.com (M.M.G.); amaninaahmadsafri@gmail.com (A.A.S.); \\ jafferu@gmail.com (U.J.) \\ 2 Department of Haematology, School of Medical Sciences, Universiti Sains Malaysia, Kubang Kerian 16150, \\ Kelantan, Malaysia; wzaidah@usm.my \\ 3 Hospital Universiti Sains Malaysia, Jalan Raja Perempuan Zainab II, Kubang Kerian 16150, Kelantan, \\ Malaysia; nursuhaila@usm.my \\ 4 Department of Family Medicine, School of Medical Sciences, Universiti Sains Malaysia, Kubang Kerian 16150, \\ Kelantan, Malaysia \\ * Correspondence: mmuzaimi@usm.my
}

check for updates

Citation: Che Mohd Nassir, C.M.N.; Mohamad Ghazali, M.; Ahmad Safri, A.; Jaffer, U.; Abdullah, W.Z.; Idris, N.S.; Muzaimi, M. Elevated Circulating Microparticle Subpopulations in Incidental Cerebral White Matter Hyperintensities: A Multimodal Study. Brain Sci. 2021, 11, 133. https://doi.org/10.3390/brainsci 11020133

Received: 9 December 2020 Accepted: 19 January 2021 Published: 20 January 2021

Publisher's Note: MDPI stays neutral with regard to jurisdictional claims in published maps and institutional affiliations.

Copyright: (c) 2021 by the authors. Licensee MDPI, Basel, Switzerland. This article is an open access article distributed under the terms and conditions of the Creative Commons Attribution (CC BY) license (https:/ / creativecommons.org/licenses/by/ $4.0 /)$.

\begin{abstract}
Asymptomatic (or "silent") manifestations of cerebral small vessel disease (CSVD) are widely recognized through incidental findings of white matter hyperintensities (WMHs) as a result of magnetic resonance imaging (MRI). This study aims to examine the potential associations of surrogate markers for the evaluation of white matter integrity in CSVD among asymptomatic individuals through a battery of profiling involving QRISK2 cardiocerebrovascular risk prediction, neuroimaging, neurocognitive evaluation, and microparticles (MPs) titers. Sixty asymptomatic subjects (mean age: $39.83 \pm 11.50$ years) with low to moderate QRISK2 scores were recruited and underwent neurocognitive evaluation for memory and cognitive performance, peripheral venous blood collection for enumeration of selected MPs subpopulations, and 3T MRI brain scan with specific diffusion MRI (dMRI) sequences inclusive of diffusion tensor imaging (DTI). WMHs were detected in 20 subjects (33\%). Older subjects (mean age: $46.00 \pm 12.00$ years) had higher WMHs prevalence, associated with higher QRISK2 score and reduced processing speed. They also had significantly higher mean percentage of platelet (CD62P)- and leukocyte (CD62L)-derived MPs. No association was found between reduced white matter integrity-especially at the left superior longitudinal fasciculus (LSLF) — with age and neurocognitive function; however, LSLF was associated with higher QRISK2 score, total MPs, and CD62L- and endothelial cell-derived MPs (CD146). Therefore, this study establishes these multimodal associations as potential surrogate markers for "silent" CSVD manifestations in the well-characterized cardiocerebrovascular demographic of relatively young, neurologically asymptomatic adults. Furthermore, to the best of our knowledge, this study is the first to exhibit elevated MP counts in asymptomatic CSVD (i.e., CD62P and CD62L), which warrants further delineation.
\end{abstract}

Keywords: cerebral small vessel disease; cardiocerebrovascular; microparticles; diffusion MRI

\section{Introduction}

Cerebral small vessel disease (CSVD) consists of a spectrum of histopathological, clinical, and imaging abnormalities linked to pathology in the microvasculature of small penetrating arteries and arterioles (50-500 $\mu \mathrm{m})$ in brain irrigating subcortical structures [1-3]. Generally, it results in brain parenchyma injuries, which are invariably associated with distal leptomeningeal and intracerebral vessel pathology, affecting deep cerebral white 
matter integrity [4]. Current data suggest that CSVD is one of the most prevalent neurological disorders in aging societies of the developed world $[5,6]$. The prevalence of apparently asymptomatic manifestations of CSVD, such as silent brain infarcts or white matter hyperintensities (WMHs), has been associated with advancing age [7].

"Silent" or "asymptomatic" manifestations of CSVD are frequently detected as an incidental finding from brain imaging and are often used as a prognosis marker following a first symptomatic CSVD presentation [8]. In magnetic resonance imaging (MRI), the micro-changes in white matter can be seen as WMHs that represent more diffuse areas of white matter lesions [9]. Such changes, as seen on a conventional MRI, have shown limited correlation with clinical parameters (e.g., cognition), ranging from weak to moderate [10]. Thus, more concise correlations and reliable lesion surrogates are required in order to enhance the evaluation of white matter structure integrity, including white matter tractography. Advancements in diffusion MRI (dMRI) techniques have offered potential new information on disease pathomechanisms as well as surrogate markers for disease onset and progression, and new therapy evaluations using newer magnetic resonance (MR)-based diffusion tensor imaging (DTI) modalities [11,12].

Furthermore, as part of risk evaluation, essential clinical algorithms have become useful in the prevention and management of CSVD, including cerebrovascular events [13]. In this line, QRISK has been developed to evaluate cardiovascular risk as well as the risk of cerebrovascular diseases such as CSVD. QRISK is an online-based tool that predicts cardiocerebrovascular disease risk over the next 10 years [14]. However, the correlation with MR-DTI findings in white matter changes using QRISK and its neurocognitive evaluations performance among asymptomatic individuals are still insufficient. Moreover, at present, systematic data on CSVD are nonexistent in Malaysia.

Microparticles (MPs) are non-nucleated, small (0.1-1 $\mu \mathrm{m}$ in diameter), and membraneenclosed extracellular particles [15-17], which are formed through various triggered mechanisms-including cellular activation, injury, or apoptosis-which cause membrane phospholipids exocytic blebs to be released from the cell surface [18-20]. MPs compositions are heterogeneous and can originate from different cell types, such as platelets, leukocytes, erythrocytes, and endothelial cells $[21,22]$. They inherit their parental cell surface proteins and cytoplasmic materials [23]. Their formation and accumulation have been reported to affect the proper functioning of small vessels. A previous study has shown that endothelial dysfunction caused by MPs can induce vascular inflammation, potentially leading to a prothrombotic state in arteriosclerosis and atherosclerosis [22]. Furthermore, MPs shedding has been implicated in ischemic stroke subtypes [24]. In CSVD, such a manifestation is related to the disorganization of arterial segmental walls and luminal narrowing, where the accumulation of MPs (alongside cholesterol crystals) may trigger hypoperfusion, leading to microinfarcts and WMHs $[25,26]$.

Furthermore, variations in MPs and their subpopulation counts in various diseases have indicated their diagnostic importance, particularly in vascular pathologies. However, most of the previous studies regarding MPs have been skewed toward disease populations and did not focus on asymptomatic subjects with a prevalence of WMHs. Hence, in the present study, we sought to find novel correlations and associations of MPs and their subpopulation counts with an imaging modality, QRISK scores, and neurocognitive profiles as potential surrogate markers for CSVD among asymptomatic individuals with wellcharacterized cardiovascular demographics in suburban southeast Peninsular Malaysia.

\section{Materials and Methods}

\subsection{Ethics Approval, Sample Size Estimation, and Subject Recruitment}

The present study was conducted at the Hospital Universiti Sains Malaysia, a suburban tertiary referral center for neurological disorders on the east coast of Peninsular Malaysia, with a catchment population base of over 4 million people. Ethics approval was obtained from the Human Research Ethics Committee at Universiti Sains Malaysia (USM/JEPeM/15030096). Subjects were recruited through simple convenience random 
sampling (among those who attended the Family Medicine Clinic at HUSM for their routine medical follow-up appointments). Demographic (i.e., age, sex, and race) and clinical (i.e., smoking status, diabetes, body mass index, and systolic blood pressure) information were collected before cardiocerebrovascular risk calculation using QRISK2. The QRISK2 version 2018 (http:/ / www.QRISK.org/index.php; the University of Nottingham and EMIS), an online-based cardiocerebrovascular calculator, was used to calculate the percentage of disease risk (over the next 10 years) for all recruited subjects. The projected risk for the subjects was arranged according to the following criteria: 0 to $10 \%=$ no/low cardiovascular risk; 10.1 to $20 \%=$ moderate risk; and $\geq 20.1 \%$ = high risk [13]. Asymptomatic subjects aged 25-62 years without any medical symptoms and no history of neurological diseases as well as "no/low to moderate cardiovascular risk" were included in the present study. Subjects with "high cardiocerebrovascular risk" percentage and/or with a previous history of neurological diseases were excluded.

\subsection{Neurocognitive Assessment}

The Wechsler Adult Intelligence Scale: Fourth edition (WAIS-IV) was used to evaluate the general cognitive and memory abilities of the subjects. The perceptual reasoning index (PRI) from WAIS-IV was used to calculate nonverbal reasoning and perceptual organization through three subtests: block design, matrix reasoning, and visual puzzle. The working memory index (WMI) was used to specifically measure simultaneous and sequential processing, attention, and concentration through two subtests: digit span and letter-number sequencing. Finally, the processing speed index (PSI) was used to calculate the speed of mental and graphomotor processing through two subtests: coding and symbol search. Scores obtained from each subtest were used for the multimodal comparison.

\subsection{MPs Enumeration}

A total of $18 \mathrm{~mL}$ of fasting peripheral venous blood samples was collected from each subject using BD Vacutainer ${ }^{\circledR}$ tubes (Becton Dickinson, BD, Plymouth, UK) containing active anticoagulant trisodium citrate with citric acid and dextrose (solution B). A multisample Luer Adaptor (Terumo, Malaysia) and 21-gauge needles (Terumo Corporation, Biñan, Philippines) were used to aid in blood drawing. To minimize platelet activation during blood sampling, no tourniquets were used. As much as possible, MPs generation was prevented by transporting the tubes with minimal agitation and by keeping them at $25^{\circ} \mathrm{C}$, following previously published recommendations [27].

The blood samples were centrifuged at $2500 \times g$ for $15 \mathrm{~min}$ at $24{ }^{\circ} \mathrm{C}$ to separate the plasma from blood cells and platelets [28]. The platelet-poor plasma was separated through the second centrifugation step $\left(2000 \times g, 10 \mathrm{~min}, 24^{\circ} \mathrm{C}\right)$, in order to obtain platelet-free plasma (PFP). A third centrifugation step $\left(2000 \times g, 10 \mathrm{~min}, 24^{\circ} \mathrm{C}\right)$ was carried out to better separate the PFP. Enumeration of MPs was performed directly in fresh PFP in order to avoid MPs loss and to preserve MPs characteristics [27]. The unused test samples were kept in a $-80^{\circ} \mathrm{C}$ freezer.

Before flow cytometry analysis, $50 \mu \mathrm{L}$ of freshly isolated PFP was incubated in the dark for 20 min at $4{ }^{\circ} \mathrm{C}$ after being diluted with $50 \mu \mathrm{L}$ of $0.5 \times$ Annexin- $V$ binding buffer (Becton Dickinson (BD), Bioscience, USA), followed by staining with fluorescein isothiocyanate Annexin-V ( $2 \mu \mathrm{L}$, Becton Dickinson (BD), Bioscience, Franklin Lakes, NJ, USA) and fluorescence-conjugated monoclonal antibodies: APC CD62P (platelet-derived MPs; $2 \mu \mathrm{L}$, Becton Dickinson (BD), Bioscience, USA), PE-Cy 7 mouse anti-human CD235a (erythrocytesderived MPs; $2 \mu \mathrm{L}$, Becton Dickinson (BD), Bioscience, USA), PE CD62L (leucocytes-derived MPs; $2 \mu \mathrm{L}$, Becton Dickinson (BD), Bioscience, USA), and PE-Cy7 mouse anti-human CD146 (endothelial cell-derived MPs; $2 \mu \mathrm{L}$, Becton Dickinson (BD), Bioscience, USA).

Thereafter, $350 \mu \mathrm{L}$ of $1 \times$ Annexin-V binding buffer was added to the samples. They were then shaken gently and analyzed by flow cytometry assay (FACS Canto II, Becton Dickinson, BD Biosciences, USA) at a low-rate setting. All MPs were measured using both forward scatter and side scatter. The gating mechanism was based on previous 
studies [18,29-31]. We defined MPs, for the purposes of the present study, as particles that have a diameter less than $1.0 \mu \mathrm{m}$. Thus, MPs that had positive staining for Annexin-V and expressed specific markers were separated as true events from background noise. The samples were run for flow analyzed at rates below 10,000 events/s and stopped when 10,000 events within the MPs gate were collected or stopped after a fixed time of $5 \mathrm{~min}$. The MPs subpopulation counts were calculated as the number of events per MPs gate, whereas total MPs count was calculated by a summation of multiple MPs subpopulation counts.

\subsection{Diffusion MRI Protocols}

A Philips Achieva (Best, The Netherlands) 3T MRI machine with a 32-channel head coil ( $b$-value: $\left.1000 \mathrm{~s} / \mathrm{mm}^{2}\right)$ was used for brain scanning with the following acquisition parameters: For structural image acquisition 3D-T1, echo time (TE)/repetition time $(\mathrm{TR})=10 / 678 \mathrm{~ms}$, reconstruction matrix $=512 \times 512 \times 40$, field of view $(\mathrm{FOV})=230 \mathrm{~mm}$, voxel size $=0.45 \mathrm{~mm} \times 0.45 \mathrm{~mm}$, slice spacing $=0 \mathrm{~mm}$, slice thickness $=2.5 \mathrm{~mm}$, flip angle $=70^{\circ}$, and 180 contiguous sagittal slices orientation; for T2, TE $/ \mathrm{TR}=80 / 3000 \mathrm{~ms}$, reconstruction matrix $=512 \times 512 \times 24, \mathrm{FOV}=230 \mathrm{~mm}$, voxel size $=0.45 \times 0.45$, slice spacing $=1.0 \mathrm{~mm}$, slice thickness $=2.5 \mathrm{~mm}$, and flip angle $=90^{\circ}$; for 3D-fluid attenuated inversion recovery (FLAIR), TE $/ \mathrm{TR}=125 / 11,000 \mathrm{~ms}, \mathrm{TI}=2800 \mathrm{~ms}$, reconstruction matrix $=512 \times 512 \times 24, \mathrm{FOV}=230 \mathrm{~mm}$, voxel size $=0.45 \mathrm{~mm} \times 0.45 \mathrm{~mm}$, slice spacing $=0 \mathrm{~mm}$, slice thickness $=2.5 \mathrm{~mm}$, flip angle $=90^{\circ}$, and 170 contiguous sagittal slices orientation; and for DTI, a scheme of 32 directions with a b-value of $1000 \mathrm{~s} / \mathrm{mm}^{2}, \mathrm{TE} / \mathrm{TR}=72 / 6951 \mathrm{~ms}$, reconstruction matrix $=96 \times 96, \mathrm{FOV}=240 \mathrm{~mm}$, voxel sizes $=2.5 \mathrm{~mm} \times 2.5 \mathrm{~mm}$, slice spacing $=0 \mathrm{~mm}$, slice thickness $=2.5 \mathrm{~mm}$, and flip angle $90^{\circ}$. The total scanner time was 15-20 $\mathrm{min}$, per the subject's ability.

\subsection{Image Analysis}

Images were first visualized by an assigned radiologist using the MeDINria version 2.2 software [32] in order to manually detect the presence of WMHs. The severity was evaluated using the Fazekas scale $[33,34]$. The presence of WMHs was further confirmed using the white matter lesion segmentation technique. This procedure was used to automatically detect and evaluate WMHs. To do this, lesion segmentation tools (LSTs) were used. The LST was evaluated in functional MRI Toolbox of Statistical Parametric Mapping version 12 (Institute of Neurology, University College London, London, UK) and installed through the Matrix Laboratory (MATLAB) version 2017a software (The MathWorks, Natick, MA, USA).

The DSI studio software (http:/ / dsi-studio.labsolver.org) was used for image processing in white matter tractography for subjects with $\mathrm{WMHs}\left(\mathrm{WMH}^{+}\right)$and those without WMHs $\left(\mathrm{WMH}^{-}\right)$. In DSI studio, a DTI diffusion scheme was used for image reconstruction and tensor estimation. A total of 32 diffusion sampling directions were acquired. The $b$ value was $1000 \mathrm{~s} / \mathrm{mm}^{2}$. The in-plane resolution was $2.5 \mathrm{~mm}$. The slice thickness was $2.5 \mathrm{~mm}$. The diffusion tensor was calculated.

\subsection{Region of Interest Analysis and Tractography}

With the anatomical reference from FLAIR imagery, a voxel-based region of interest (ROI) identification technique was applied over the selected WMHs in the axial plane, followed by regional tractography. A deterministic fiber tracking algorithm was used, as previously described [35]. The seeding region was placed over the whole brain. An ROI was placed at the region where the WMHs were located. The angular threshold was $60^{\circ}$, and the step size was $1.25 \mathrm{~mm}$. The anisotropy threshold was determined automatically by DSI studio. Tracks with length less than $30 \mathrm{~mm}$ were discarded. A total of 5000 seeds were placed. Thereafter, the structure of the white matter tracts where the ROI was located was defined using a white matter atlas (John Hopkins University DTI-based White Matter Atlases, provided by DSI studio). Hence, the structures of the white matter tracts involved 
in WMHs were identified. Furthermore, the ROI for $\mathrm{WMH}^{-}$subjects was guided by the white matter tracts involved in $\mathrm{WMHs}$ first identified in $\mathrm{WMH}^{+}$subjects. The atlas of white matter tracts identified was set as a benchmark for parameter comparison between $\mathrm{WMH}^{-}$ and $\mathrm{WMH}^{+}$. Hence, the comparison between $\mathrm{WMH}^{+}$and $\mathrm{WMH}^{-}$was performed based on structures of the white matter tracts involved rather than on specific ROIs.

\subsection{Statistical Data Analysis}

The collected data were analyzed using the Statistical Package for Social Sciences version 24 software (IBM Corp., Armonk, NY, USA). Alpha ( $\alpha$ ) was set at 0.05 , and for all analyses, $p>0.05$ was considered statistically significant with a confidence interval of $95 \%$. Descriptive statistical analysis, such as mean and standard deviation, was applied to all data. Correlations between variables were obtained using Pearson's correlation coefficient. Mean differences between and within variables were obtained using an independent sample T-test for significant normally distributed data; meanwhile, the Mann-Whitney $\mathrm{U}$ test was used for nonnormally distributed data. A linear regression model was used to study the associations between age, QRISK2 score, neurocognitive function, and MPs. These analyses were adjusted for gender, ethnicity, and prior cardiocerebrovascular disease risk factors. Subsequent analyses were conducted in order to investigate the associations of DTI parameters (i.e., tract specific integrity), adjusted for age, QRISK2 score, neurocognitive function, and MPs subpopulations.

\section{Results}

\subsection{Demographics and Cardiocerebrovascular Risk Prediction}

Sixty subjects (mean age: $39.83 \pm 11.50$ years) who fulfilled the inclusion and exclusion criteria for the sample were recruited. The majority were female, Malay ethnicity, nonsmokers, and non-hypertensive (Table 1). Based on the QRISK2 profiles, the overall mean percentage of subjects that will develop cardiocerebrovascular risk for the next 10 years was $2.83 \pm 4.42 \%$. Furthermore, all subjects were at no/low-moderate risk, signifying that the subjects of the present study were potentially normal/healthy, with low or no chance of developing vascular diseases (i.e., asymptomatic). Older subjects ( $\geq 40$ years old; mean QRISK2: $3.99 \pm 3.75 \%)$ had a significantly higher QRISK2 percentage $(p=0.00<0.05)$ than younger subjects ( $\leq 39$ years old; mean QRISK2: $0.34 \pm 0.29 \%$ ). Hence, the mean QRISK2 of older subjects was higher than that of the younger subjects.

Table 1. Distribution of demographics variables in the study $(N=60$, mean age $=39.83 \pm 11.50$ years).

\begin{tabular}{|c|c|c|}
\hline & Variables & $n(\%)$ \\
\hline \multirow{4}{*}{ Age } & $<20$ & $0(0)$ \\
\hline & $21-40$ & 31 (51.7) \\
\hline & $41-60$ & $26(43.3)$ \\
\hline & $>60$ & $3(5)$ \\
\hline \multirow{2}{*}{ Gender } & Male & $19(31.7)$ \\
\hline & Female & $41(68.3)$ \\
\hline \multirow{3}{*}{ Ethnic } & Malay & $54(90.0)$ \\
\hline & Chinese & $4(6.7)$ \\
\hline & Other & $2(3.3)$ \\
\hline \multirow{2}{*}{ WMHs * } & Absent $\left(\mathrm{WMH}^{-}\right)$ & $40(66.7)$ \\
\hline & Present $\left(\mathrm{WMH}^{+}\right)$ & $20(33.3)$ \\
\hline
\end{tabular}


Table 1. Cont.

\begin{tabular}{ccc}
\hline & Variables & $\boldsymbol{n} \mathbf{( \% )}$ \\
\hline \multirow{3}{*}{ Smoking status } & Nonsmoker & $52(86.7)$ \\
& Ex-smoker & $7(11.7)$ \\
Family history of coronary heart disease in & Light smoker & $1(1.7)$ \\
1st degree relative under 60 years old & Yes & $15(25.0)$ \\
\hline Treated Hypertension & No & $45(75.0)$ \\
\hline Atrial fibrillation & Yes & $9(15.0)$ \\
\hline Type 2 Diabetes & No & $51(85.0)$ \\
\hline Hyperlipidemia & Yes & - \\
& No & $60(100)$ \\
\hline \multirow{2}{*}{ Chronic kidney disease (stage 4 or 5) } & Yes & - \\
& No & - \\
\hline \multirow{2}{*}{ T) } & Yes & $60(100)$ \\
\hline
\end{tabular}

*WMHs, white matter hyperintensities.

\subsection{Age vs. the Proportion of WMHs among the Study Subjects}

Among the 60 subjects, 20 (33\%) were identified as $\mathrm{WMH}^{+}$(mean age: $46.00 \pm 12.00$ years) with grade 1 Fazekas (see Figure 1 ) and the rest were identified as $\mathrm{WMH}^{-}$(mean age: $36.75 \pm 10.04$ years). Among the $\mathrm{WMH}^{+}$subjects, $14(70 \%)$ were among the older subjects (mean age: $49.70 \pm 7.95$ years). There was a significant age difference between $\mathrm{WMH}^{-}$and $\mathrm{WMH}^{+}$subjects (see Table 2). Therefore, in the present study, WMHs were more prevalent in older individuals. Nevertheless, we also found WMHs among the younger subjects (mean age: $36.75 \pm 10.04$ years).

Table 2. Independent $T$-tests for comparison of the study variables between $\mathrm{WMH}^{+}$and $\mathrm{WMH}^{-}$ subjects.

\begin{tabular}{ccccc}
\hline & \multicolumn{2}{c}{ Mean \pm SD } & \\
\cline { 2 - 3 } Variables & $\begin{array}{c}\mathbf{W M H}^{-} \\
(\boldsymbol{n}=\mathbf{2 0})\end{array}$ & $\begin{array}{c}\mathbf{W M H}^{+} \\
(\boldsymbol{n = 4 0 )}\end{array}$ & T-Statistics $(\boldsymbol{d} \boldsymbol{f})$ & $p$-Value \\
& $36.75 \pm 10.04$ & $46.00 \pm 12.00$ & $-2.98(32.66)$ & $0.006^{*}$ \\
Age & $1.32 \pm 1.54$ & $5.84 \pm 6.43$ & $-4.24(58)$ & $0.000^{*}$ \\
QRISK2 & $102.95 \pm 13.30$ & $101.30 \pm 10.73$ & $0.52(46.12)$ & 0.607 \\
PRI & $108.53 \pm 19.14$ & $105.25 \pm 14.55$ & $0.74(48.45)$ & 0.465 \\
WMI & $103.73 \pm 14.31$ & $98.05 \pm 11.49$ & $1.66(46.33)$ & 0.104 \\
PSI & $98.30 \pm 42.43$ & $163.85 \pm 140.72$ & $-2.73(58)$ & $0.008^{*}$ \\
CD62L & $33.05 \pm 74.25$ & $50.45 \pm 42.10$ & $-1.16(56.95)$ & 0.252 \\
CD235a & $150.45 \pm 75.21$ & $370.80 \pm 330.271$ & $-4.05(58)$ & $0.000^{*}$ \\
CD62P & $25.08 \pm 20.27$ & $194.80 \pm 669.90$ & $-1.62(58)$ & 0.112 \\
CD146 & $306.88 \pm 152.15$ & $779.90 \pm 930.04$ & $-3.16(58)$ & $0.003^{*}$ \\
Total MPs &
\end{tabular}

Notes: WMH, white matter hyperintensities; SD, standard deviation; df, degree of freedom; PRI, perceptual reasoning index; WMI, working memory index; PSI, processing speed index; CD, cluster differentiations; MPs, microparticles. ${ }^{*} p=$ significant difference (1-tailed) at the 0.01 level. 


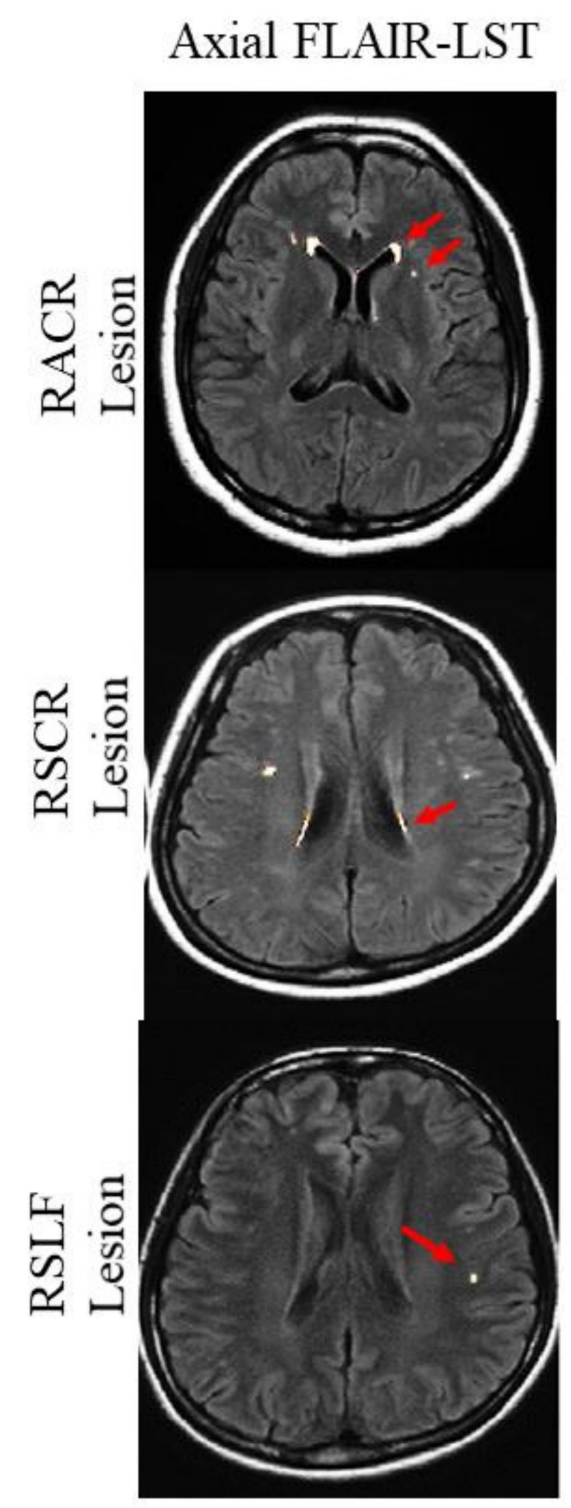

\section{ROI-White Matter Tractography} Axial Coronal
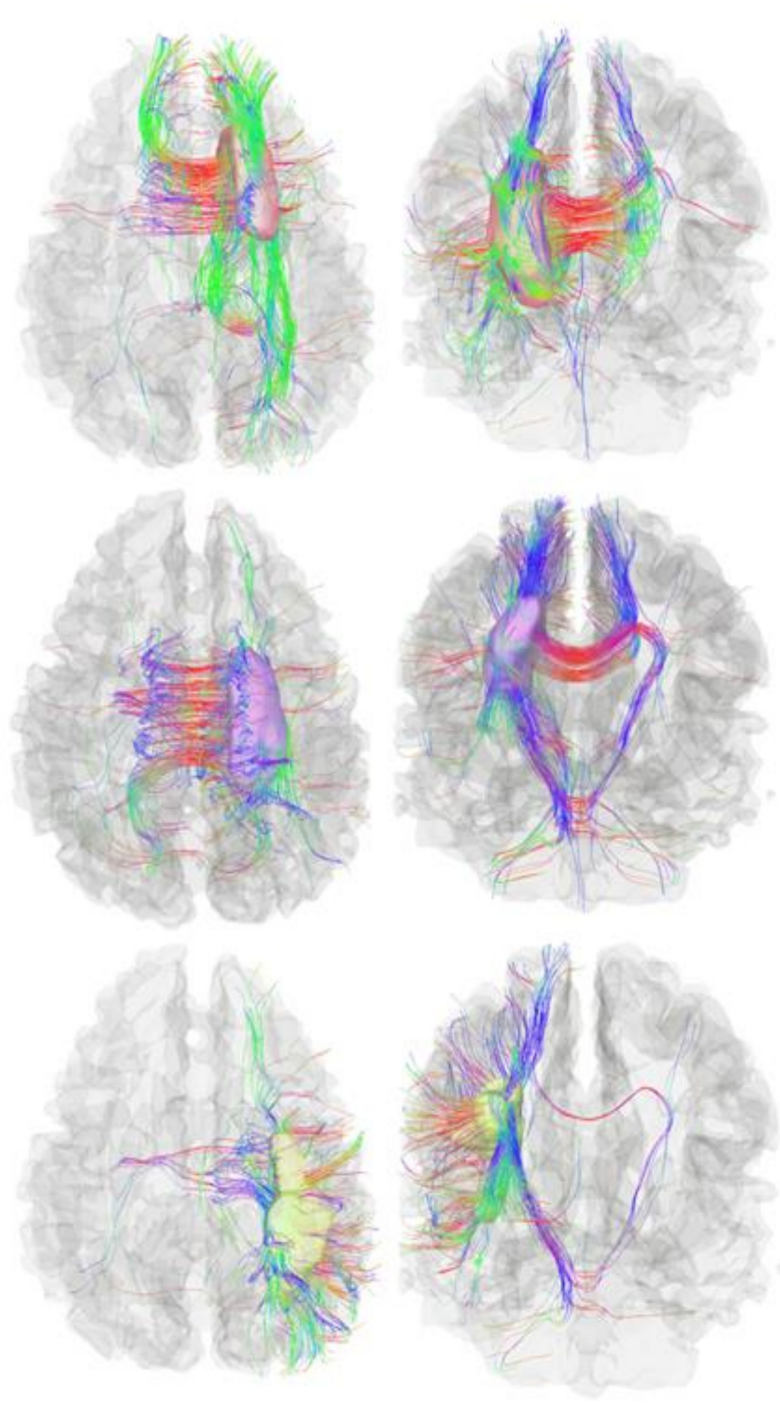

Figure 1. From left: the first row shows fluid attenuated inversion recovery (FLAIR) images with white matter hyperintensities (WMHs) voxel outputs from the lesion segmentation tool (LST) showing right anterior corona radiata (RACR) lesion (red arrow) and RACR tractography (axial and coronal view). The second row shows FLAIR images with WMHs voxel outputs from the LST showing right superior corona radiata (RSCR) lesion (red arrow) and RSCR tractography (axial and coronal view). The third row shows FLAIR images with WMHs voxel outputs from the LST showing right superior longitudinal fasciculus (RSLF) lesion (red arrow) and RSLF tractography (axial and coronal view). The blue, red, and green colors on the right figures represent white matter fibers running along the inferior-superior, right-left, and anterior-posterior orientations, respectively. Notes: LST followed the native Statistical Parametric Mapping (SPM) and Matrix Laboratory (MATLAB) orientations for producing JPEG images; hence, the original radiological view (left on right) obtained from the magnetic resonance imaging (MRI) scanner was shifted to neurological view (right on right) as a reminder of viewing interpretation. FLAIR, fluid-attenuated inverse recovery; RACR, right anterior corona radiata; RSLF, right superior longitudinal fasciculus; RSCR, right superior corona radiata; ROI, region of interest.

\subsection{QRISK2 vs. the Proportion of WMHs among the Study Subjects}

We found that $\mathrm{WMH}^{+}$subjects had a significantly higher mean percentage of QRISK2 than $\mathrm{WMH}^{-}$subjects (Table 2). In the present study, the prevalence of WMHs was higher 
in older subjects; in the same line, QRISK2 has a stronger correlation with age, as shown by the linear model (Table 3).

Table 3. Correlation and linear regression profile of study variables.

\begin{tabular}{|c|c|c|c|c|c|c|}
\hline \multirow{2}{*}{ No. } & \multirow{2}{*}{ Variables } & \multicolumn{5}{|c|}{$r(\beta)$} \\
\hline & & 1 & 2 & 3 & 4 & 5 \\
\hline 1 & Age & 1 & & & & \\
\hline 2 & QRISK2 & $0.75^{*}\left(0.57^{*}\right)$ & 1 & & & \\
\hline 3 & PRI & $-0.27 *(0.02)$ & $-0.23 *(-0.09)$ & 1 & & \\
\hline 4 & WMI & $-0.35 *(-0.01)$ & $-0.32 *(-0.07)$ & $0.62 *(0.35 *)$ & 1 & \\
\hline 5 & PSI & $-0.56^{*}\left(-0.31^{*}\right)$ & $-0.40 *(-0.19)$ & $0.60 *(0.44 *)$ & $0.61 *(0.63 *)$ & 1 \\
\hline 6 & CD62L & $0.51 *(0.19)$ & $0.58 *(0.41 *)$ & $0.09(0.25 *)$ & $-0.12(0.12)$ & $-0.19(-0.18)$ \\
\hline 7 & CD235a & $0.26 *(0.18)$ & $0.13(0.02)$ & $0.10(0.11)$ & $0.05(0.19)$ & $-0.16(-0.15)$ \\
\hline 8 & CD62P & $0.32 *(0.16)$ & $0.44 *(-0.08)$ & $0.08(0.32)$ & $-0.15(-0.09)$ & $-0.15(0.01)$ \\
\hline 9 & CD146 & $0.26 *(0.14)$ & $0.41 *(0.28)$ & $-0.18(-0.16)$ & $-0.14(-0.42)$ & $-0.07(0.05)$ \\
\hline 10 & Total MPs & $0.40 *(-0.02)$ & $0.54 *(0.30)$ & $-0.06\left(-0.34^{*}\right)$ & $-0.16(-0.12)$ & $-0.15(0.00)$ \\
\hline
\end{tabular}

Notes: $r=$ Pearson correlation coefficient, $\beta=$ standardized beta coefficients; PRI, perceptual reasoning index; WMI, working memory index; PSI, processing speed index; $\mathrm{CD}$, cluster differentiations; MPs, microparticles. * $p=$ significant difference (1-tailed) at the 0.01 level.

\subsection{Neurocognitive Profiles vs. the Proportion of WMHs among the Study Subjects}

We found that $\mathrm{WMH}^{-}$subjects had higher mean scores of PRI, WMI, and PSI than $\mathrm{WMH}^{+}$subjects, although the differences were not significant (see Table 2). Furthermore, we also found that age and QRISK2 had negative correlations with the neurocognitive performance of the study subjects. However, the linear model only revealed a significant relationship between age and PSI (Table 3). Therefore, it seems that processing speed is reduced with advancing age, especially in asymptomatic individuals with WMHs.

\subsection{MPs vs. the Proportion of WMHs among the Study Subjects}

We found that $\mathrm{WMH}^{+}$subjects had higher individual MPs counts than $\mathrm{WMH}^{-}$subjects. The differences were significant only for leukocyte-derived MPs (CD62L) and plateletderived MPs (CD62P) but not for red blood cell-derived MPs (CD235a) and endothelial cell-derived MPs (CD146) (Table 2). Total MPs counts were also significantly higher in $\mathrm{WMH}^{+}$subjects. We also found a weak to moderate positive correlation between the age and QRISK2 of subjects with MPs counts (Table 3). The strongest correlation was between CD62L with age and QRISK2. However, the linear model showed that CD62L was significantly associated with QRISK2 only, not with age. Furthermore, no significant correlation was found between MPs counts and neurocognitive profiles (PRI, WMI, or PSI). It is of interest that CD62L and total MPs count appeared to be significantly associated only with PRI and not with WMI or PSI performances.

\subsection{Multimodal Study for Cerebral White Matter Integrity among the Study Subjects}

Three bilateral white matter tracts (bilateral anterior corona radiata, ACR; superior corona radiata, SCR; and superior longitudinal fasciculus, SLF) were chosen based on the prevalence of WMHs (location-wise), as seen in the MRI brain images of the study subjects. Therefore, six ROIs were selected, regarding the presence of WMHs in specific tracts: right ACR (RACR) $(n=7)$, left ACR (LACR) $(n=7)$, right SCR (RSCR) $(n=4)$, left SCR (LSCR) $(n=4)$, right SLF (RSLF) $(n=5)$, and left SLF (LSLF) $(n=3)$ (see Figure 1). White matter integrity was defined by the value of diffusion parameters of certain white matter tracts, such as (1) fractional anisotropy (FA), where lower FA signifies reduced white matter integrity due to reduced restriction in molecular motion following disruption in axonal cell membranes and myelin sheathes [36,37]; (2) mean diffusivity (MD), where increased MD also signifies reduced white matter integrity following an increase in mean molecular motion concerning disruption in cellular properties (i.e., integrity and 
size) [37,38]; and (3) radial diffusivity (RD) and axial diffusivity (AD), defined by the rate of diffusion along cellular axes [39], where RD/AD varies across brain regions [40].

We found that, compared with $\mathrm{WMH}^{-}$subjects, $\mathrm{WMH}^{+}$subjects had slightly lower FA, higher MD, and various ADs/RDs in all selected tracts. However, as shown in Table 4, the differences were not significant. Furthermore, FA had a significant negative correlation (low to moderate) with age and QRISK2, whereas AD had a significant negative correlation (low) with age only (Table 5). Further analysis, however, showed no association between FA, MD, AD, and RD with age and QRISK2. Hence, advancing age and percentage of QRISK2 do not seem to serve as direct measures of white matter integrity for the selected tracts in the present study. However, the linear model revealed a significant association between QRISK2 and the FA, MD, AD, and RD parameters of the LSLF tract. Hence, it can be postulated that a higher QRISK2 can predict a reduction in LSLF integrity (lower FA and higher MD; see Table 5).

Table 4. Comparison of the integrity of selected white matter between $\mathrm{WMH}^{+}$and $\mathrm{WMH}^{-}$subjects with independent $T$-tests.

\begin{tabular}{|c|c|c|c|c|}
\hline \multirow{2}{*}{ Tracts Parameters } & \multicolumn{2}{|c|}{$($ Mean \pm SD) } & \multirow{2}{*}{$T$-Statistics $(d f)$} & \multirow{2}{*}{$p$-Value } \\
\hline & WMH $^{-}$ & $\mathrm{WMH}^{+}$ & & \\
\hline \multicolumn{5}{|l|}{ RACR } \\
\hline FA & $0.42 \pm 0.03$ & $0.42 \pm 0.03$ & 0.35 (37.94) & 0.727 \\
\hline MD & $0.82 \pm 0.07$ & $0.84 \pm 0.08$ & $-0.59(33.83)$ & 0.560 \\
\hline $\mathrm{AD}$ & $1.23 \pm 0.13$ & $1.24 \pm 0.14$ & $-0.34(35.39)$ & 0.733 \\
\hline $\mathrm{RD}$ & $0.63 \pm 0.05$ & $0.64 \pm 0.06$ & $-0.86(32.21)$ & 0.395 \\
\hline \multicolumn{5}{|l|}{ LACR } \\
\hline FA & $0.42 \pm 0.02$ & $0.42 \pm 0.27$ & $0.45(34.42)$ & 0.649 \\
\hline MD & $0.82 \pm 0.08$ & $0.83 \pm 0.08$ & $-0.24(38.36)$ & 0.812 \\
\hline $\mathrm{AD}$ & $1.23 \pm 0.13$ & $1.23 \pm 0.13$ & $-0.05(38.38)$ & 0.957 \\
\hline RD & $0.62 \pm 0.05$ & $0.63 \pm 0.05$ & $-0.46(37.81)$ & 0.645 \\
\hline \multicolumn{5}{|l|}{ RSCR } \\
\hline FA & $0.46 \pm 0.03$ & $0.46 \pm 0.02$ & $-0.06(48.00)$ & 0.955 \\
\hline MD & $0.81 \pm 0.07$ & $0.81 \pm 0.07$ & $-0.05(37.40)$ & 0.958 \\
\hline $\mathrm{AD}$ & $1.26 \pm 0.13$ & $1.26 \pm 0.12$ & $-0.02(40.09)$ & 0.987 \\
\hline $\mathrm{RD}$ & $0.59 \pm 0.04$ & $0.59 \pm 0.05$ & $-0.09(34.21)$ & 0.923 \\
\hline \multicolumn{5}{|l|}{ LSCR } \\
\hline FA & $0.46 \pm 0.02$ & $0.46 \pm 0.02$ & 0.17 (41.85) & 0.866 \\
\hline MD & $0.82 \pm 0.07$ & $0.83 \pm 0.08$ & $-0.30(34.89)$ & 0.765 \\
\hline $\mathrm{AD}$ & $1.26 \pm 0.12$ & $1.27 \pm 0.13$ & $-0.16(36.26)$ & 0.875 \\
\hline RD & $0.59 \pm 0.05$ & $0.60 \pm 0.06$ & $-0.45(33.64)$ & 0.655 \\
\hline \multicolumn{5}{|l|}{ RSLF } \\
\hline FA & $0.40 \pm 0.02$ & $0.40 \pm 0.03$ & $0.68(34.32)$ & 0.499 \\
\hline MD & $0.77 \pm 0.07$ & $0.78 \pm 0.08$ & $-0.24(34.15)$ & 0.813 \\
\hline $\mathrm{AD}$ & $1.13 \pm 0.11$ & $1.14 \pm 0.12$ & $-0.00(35.76)$ & 0.998 \\
\hline $\mathrm{RD}$ & $0.59 \pm 0.05$ & $0.60 \pm 0.06$ & $-0.49(32.15)$ & 0.627 \\
\hline \multicolumn{5}{|l|}{ LSLF } \\
\hline FA & $0.41 \pm 0.02$ & $0.41 \pm 0.03$ & $0.94(34.30)$ & 0.355 \\
\hline MD & $0.78 \pm 0.07$ & $0.78 \pm 0.08$ & $-0.13(33.43)$ & 0.897 \\
\hline $\mathrm{AD}$ & $1.15 \pm 0.11$ & $1.15 \pm 0.12$ & $0.14(34.98)$ & 0.892 \\
\hline RD & $0.59 \pm 0.05$ & $0.60 \pm 0.06$ & $-0.41(31.77)$ & 0.683 \\
\hline
\end{tabular}

Notes: AD, axial diffusivity; FA, fractional anisotropy; LACR, left anterior corona radiata; LSCR, left superior corona radiata; LSLF, left superior longitudinal fasciculus; MD, mean diffusivity; RACR, right anterior corona radiata; RD, radial diffusivity; RSCR, right superior corona radiata; RSLF, right superior longitudinal fasciculus; $\mathrm{WMH}$, white matter hyperintensity, $p=$ significant difference. 
Table 5. Correlation and multiple linear regression profile of study variables with white matter integrity.

\begin{tabular}{|c|c|c|c|c|}
\hline \multirow{2}{*}{$\begin{array}{c}\text { Variables \& } \\
\text { Tracts }\end{array}$} & \multicolumn{4}{|c|}{$r(\beta)$} \\
\hline & FA & MD & $\mathrm{AD}$ & RD \\
\hline \multicolumn{5}{|l|}{ Age } \\
\hline RACR & $-0.43 *(1.23)$ & $-0.13(-0.78)$ & $-0.23 *(-2.52)$ & $0.00(1.46)$ \\
\hline LACR & $-0.42 *(1.15)$ & $-0.14(-1.96)$ & $-0.23 *(-2.44)$ & $-0.01(2.74)$ \\
\hline RSCR & $-0.38 *(0.17)$ & $-0.11(-0.53)$ & $-0.20 *(0.43)$ & $0.03(0.16)$ \\
\hline LSCR & $-0.40 *(-0.80)$ & $-0.13(-0.26)$ & $-0.21 *(0.74)$ & $-0.02(-1.26)$ \\
\hline RSLF & $-0.46 *(-0.32)$ & $-0.14(-0.85)$ & $-0.23 *(-0.72)$ & $-0.02(0.27)$ \\
\hline LSLF & $-0.37 *(2.76)$ & $-0.16(1.18)$ & $-0.24 *(-5.73)$ & $-0.07(4.81)$ \\
\hline \multicolumn{5}{|l|}{ QRISK2 } \\
\hline RACR & $-0.28 *(3.90)$ & $0.05(-0.67)$ & $-0.04(-7.32)$ & $0.16(4.84)$ \\
\hline LACR & $-0.26 *(-1.87)$ & $0.05(-1.41)$ & $-0.03(4.26)$ & $0.15(-2.42)$ \\
\hline RSCR & $-0.24 *(0.47)$ & $0.05(1.44)$ & $-0.03(-1.86)$ & $0.16(0.63)$ \\
\hline LSCR & $-0.30 *(-0.41)$ & $0.05(1.81)$ & $-0.03(0.69)$ & $0.14(-0.97)$ \\
\hline RSLF & $-0.35 *(-2.94)$ & $0.07(-0.75)$ & $-0.02(5.14)$ & $0.18(-3.47)$ \\
\hline LSLF & $-0.35 *(4.09 *)$ & $0.06(3.07 *)$ & $-0.03(-9.09 *)$ & $0.17\left(8.11^{*}\right)$ \\
\hline \multicolumn{5}{|l|}{ PRI } \\
\hline RACR & $0.45^{*}(-3.55)$ & $0.15(-0.21)$ & $0.24 *(8.21)$ & $0.03(-4.79)$ \\
\hline LACR & $0.45 *(1.69)$ & $0.14(0.67)$ & $0.23 *(-3.15)$ & $0.03(1.75)$ \\
\hline RSCR & $0.33^{*}(2.61)$ & $0.14(-0.07)$ & $0.21 *(-7.64)$ & $0.05(4.25)$ \\
\hline LSCR & $0.39 *(-1.03)$ & $0.16(0.76)$ & $0.23 *(5.11)$ & $0.07(-2.65)$ \\
\hline RSLF & $0.45^{*}(-0.06)$ & $0.12(0.41)$ & $0.21 *(0.49)$ & $0.02(-1.47)$ \\
\hline LSLF & $0.42^{*}(0.57)$ & $0.16(1.27)$ & $0.24 *(-1.67)$ & $0.07(1.49)$ \\
\hline \multicolumn{5}{|l|}{ WMI } \\
\hline RACR & $0.28^{*}(-0.80)$ & $0.03(0.08)$ & $0.09(1.20)$ & $-0.06(0.30)$ \\
\hline LACR & $0.32 *(-0.14)$ & $0.01(0.21)$ & $0.08(2.30)$ & $-0.09(-2.20)$ \\
\hline RSCR & $0.14(-1.17)$ & $0.00(-0.92)$ & $0.05(0.44)$ & $-0.06(-0.17)$ \\
\hline LSCR & $0.25 *(1.95)$ & $0.00(0.14)$ & $0.06(-4.05)$ & $-0.07(3.13)$ \\
\hline RSLF & $0.36 *(-1.85)$ & $-0.00(-0.07)$ & $0.08(5.10)$ & $-0.11(-4.89)$ \\
\hline LSLF & $0.28 *(0.23)$ & $0.00(0.98)$ & $0.07(-0.27)$ & $-0.06(0.59)$ \\
\hline \multicolumn{5}{|l|}{ PSI } \\
\hline RACR & $0.28 *(0.49)$ & $-0.04(-0.79)$ & $0.04(-0.79)$ & $-0.14(1.47)$ \\
\hline LACR & $0.27 *(-1.68)$ & $-0.05(0.82)$ & $0.03(3.85)$ & $-0.15(-3.95)$ \\
\hline RSCR & $0.20 *(-0.73)$ & $-0.06(0.51)$ & $0.01(0.35)$ & $-0.15(0.24)$ \\
\hline LSCR & $0.31 *(2.10)$ & $-0.06(0.90)$ & $0.02(-4.71)$ & $-0.16(3.25)$ \\
\hline RSLF & $0.35 *(-0.14)$ & $-0.07(0.54)$ & $0.02(0.81)$ & $-0.18(-1.22)$ \\
\hline LSLF & $0.33 *(-2.02)$ & $-0.03(0.99)$ & $0.05(6.11)$ & $-0.13(-4.10)$ \\
\hline \multicolumn{5}{|l|}{ CD62L } \\
\hline RACR & $-0.03(1.36)$ & $0.17(-0.70)$ & $0.13(-1.48)$ & $0.21 *(1.05)$ \\
\hline LACR & $-0.04(-1.08)$ & $0.16(-0.89)$ & $0.12(1.42)$ & $0.21 *(-0.49)$ \\
\hline RSCR & $-0.03(-0.02)$ & $0.17(0.07)$ & $0.13(-0.46)$ & $0.22 *(0.04)$ \\
\hline LSCR & $-0.05(-0.19)$ & $0.17(0.57)$ & $0.14(1.15)$ & $0.21 *(-0.69)$ \\
\hline RSLF & $-0.08(-2.96)$ & $0.16(0.36)$ & $0.11(5.48)$ & $0.21 *(-4.42)$ \\
\hline LSLF & $-0.03\left(5.05^{*}\right)$ & $0.13(2.28 *)$ & $0.10(-11.61 *)$ & $0.16\left(9.45^{*}\right)$ \\
\hline \multicolumn{5}{|l|}{ CD235a } \\
\hline RACR & $0.00(-4.68)$ & $0.00(-0.79)$ & $-0.00(7.79)$ & $0.00(-5.04)$ \\
\hline LACR & $0.03(2.40)$ & $0.00(1.31)$ & $0.00(-2.55)$ & $0.00(2.43)$ \\
\hline RSCR & $0.00(-1.24)$ & $0.04(1.05)$ & $0.03(4.03)$ & $0.06(-1.63)$ \\
\hline LSCR & 0.07 (1.99) & $0.01(0.73)$ & $0.02(-5.03)$ & $0.01(3.15)$ \\
\hline RSLF & $-0.07(2.49)$ & $0.00(-0.45)$ & $-0.02(-7.30)$ & $0.03(4.12)$ \\
\hline LSLF & $0.06(1.15)$ & $-0.04(1.05)$ & $-0.03(-2.73)$ & $-0.05(2.07)$ \\
\hline \multicolumn{5}{|l|}{ CD62P } \\
\hline RACR & 0.10 (1.99) & $0.11(-0.06)$ & $0.11(-3.68)$ & $0.11(2.82)$ \\
\hline LACR & $0.09(-1.42)$ & $0.11(1.46)$ & $0.10(4.12)$ & $0.11(-2.47)$ \\
\hline RSCR & $0.10(-1.23)$ & $0.08(0.92)$ & $0.09(1.36)$ & $0.07(-1.98)$ \\
\hline LSCR & $0.09(2.23)$ & $0.14(-0.40)$ & $0.13(-3.38)$ & 0.15 (3.99) \\
\hline RSLF & $-0.03(-2.35)$ & $0.08(-0.01)$ & $0.06(2.97)$ & $0.12(-3.12)$ \\
\hline LSLF & $0.04(3.61)$ & $0.07(2.02)$ & $0.06(-8.37)$ & $0.08(6.87)$ \\
\hline
\end{tabular}


Table 5. Cont.

\begin{tabular}{ccccc}
\hline \multirow{2}{*}{$\begin{array}{c}\text { Variables } \& \\
\text { Tracts }\end{array}$} & FA & MD & AD & RD \\
\cline { 2 - 5 } CD146 & $0.02(2.12)$ & $0.02(0.77)$ & $0.02(-5.13)$ & $0.02(2.89)$ \\
RACR & $0.06(-2.21)$ & $0.03(1.26)$ & $0.03(6.05)$ & $0.02(-3.16)$ \\
LACR & $-0.00(2.29)$ & $0.02(1.91)$ & $0.02(-6.25)$ & $0.03(2.79)$ \\
RSCR & $0.01(-1.95)$ & $0.00(0.12)$ & $0.00(5.04)$ & $0.00(-3.31)$ \\
LSCR & $0.0(-3.16)$ & $0.06(-0.98)$ & $0.05(8.12)$ & $0.06(-4.57)$ \\
RSLF & $0.03\left(5.06^{*}\right)$ & $-0.02\left(2.26^{*}\right)$ & $-0.02(-12.01 *)$ & $-0.03\left(8.50^{*}\right)$ \\
LSLF & & & & \\
Total MPs & $0.07(2.31)$ & $0.09(0.07)$ & $0.08(-5.08)$ & $0.09(3.26)$ \\
RACR & $0.08(-2.00)$ & $0.10(1.91)$ & $0.09(5.99)$ & $0.10(-3.33)$ \\
LACR & $0.06(-0.53)$ & $0.08(1.50)$ & $0.08(0.18)$ & $0.08(-1.11)$ \\
RSCR & $0.04(1.09)$ & $0.12(-0.09)$ & $0.10(-1,67)$ & $0.13(2.23)$ \\
LSCR & $-0.04(-2.66)$ & $0.09(-0.29)$ & $0.06(4.45)$ & $0.12(-3.43)$ \\
RSLF & $0.04\left(4.82^{*}\right)$ & $0.06\left(2.77^{*}\right)$ & $0.04(-11.17 *)$ & $0.07(8.71 *)$ \\
LSLF & &
\end{tabular}

Notes: $r=$ Pearson correlation coefficient; $\beta=$ standardized beta coefficients; ${ }^{*} p=$ significant difference (1-tailed) at the $0.01 ; \mathrm{AD}$, axial diffusivity; $\mathrm{CD}$, cluster differentiation; $\mathrm{FA}$, fractional anisotropy; LACR, left anterior corona radiata; LSCR, left superior corona radiata; LSLF, left superior longitudinal fasciculus; MD, mean diffusivity; PRI, perceptual reasoning index; PSI, processing speed index; RACR, right anterior corona radiata; RD, radial diffusivity; RSCR, right superior corona radiata; RSLF, right superior longitudinal fasciculus; WMI, working memory index.

Furthermore, there was a significant positive correlation of FA in the majority of selected white matter tracts, with PRI, WMI, and PSI, despite no significant association being found (Table 5). MD and RD were not correlated with any of the cognitive indices. Meanwhile, AD was positively correlated with PRI but not with WMI and PSI, despite no significant association being found (Table 5). Hence, the scores obtained from the three cognitive indices used in the present study did not seem to be correlated with the integrity of the selected white matter tracts.

No significant correlations were found between the diffusion parameters and MP counts. However, CD62L was positively correlated with the RD of bilateral ACR, bilateral SCR, and RSLF (not LSLF), although no linear relationship was found between CD62L and $\mathrm{RD}$. It is of interest that we observed that the FA, MD, AD, and RD of LSLF had significant linear relationships with CD62L, CD146, and total MP. Hence, although the parameters were not always correlated, there is enough evidence to suggest that the enumeration of CD62L, CD146, and total MP count are associated, which may be of potential use to predict the white matter integrity of the LSLF tract.

\section{Discussion}

\subsection{Cardiocerebrovascular Risk Prediction and Age as Surrogate Markers of CSVD}

We found that $\mathrm{WMH}^{+}$subjects had higher mean QRISK2 percentage scores. Moreover, older subjects with WMHs had a significantly greater risk than younger subjects with $\mathrm{WMHs}$. Thus, $\mathrm{WMH}^{+}$subjects seem likely to show a higher risk for developing cardiocerebrovascular disease. A previous study identified aging and hypertension as major cardiovascular risk factors for the development of WMHs [40]. It has been further supported that cardiovascular risk factors and aging are the main contributors to the prevalence of WMHs and increased WMHs volume in healthy adults [41]. It has been recognized that up to $95 \%$ of elderly individuals with greater cardiovascular risk have WMHs [42], although our data comprised relatively younger adults. Furthermore, numerous studies have shown an independent association between subclinical cardiovascular risk (i.e., diabetes and hypertension) with cerebral white matter microstructure in healthy middle-to-elderly aged population [43-45].

Although most of the previous research has found a significant association between age, cardiovascular risk, and WMHs, these were derived from studies involving Caucasian 
and Afro-Caribbean populations. To date, studies on Asian populations, specifically on WMHs, remain scarce. Previous studies have reported that Asian populations (i.e., among Malays and Chinese Singaporeans) had a higher burden in cardiocerebrovascular disease, with a higher percentage of risk factors [46,47] than those reported among Caucasians [48] and, hence, a higher prevalence of WMHs [49]. Recent studies involving other Asian populations (i.e., in Singapore, Korea, and Hong Kong) have also reported aging as contributing to higher cardiovascular burden, leading to an increased prevalence of WMHs in neuroimaging [46]. It is also alarming to note the likely adverse impacts of CSVD on the quality of life and longevity among growing aging populations in Asia.

We also found that increased QRISK2 scores were significantly associated with reduced white matter integrity (in terms of lower FA and higher MD) in the LSLF tract. However, many studies have already reported that vascular risk factors, such as hypertension, smoking, and lifestyle, are associated with white matter diffusion parameters [50-53]. Although the sensitivity of diffusion parameters in detecting white matter integrity has been widely described, their complex associations with modifiable vascular risk factors remain elusive [54]. However, the association of cardiovascular risk with the integrity of specific tracts is worth considering, as the prevalence of WMHs might only affect certain tracts. From this perspective, one study has recently reported that lower FA measures in specific tracts related to WMHs in healthy middle-aged adults have a statistically significant association with the presence of multiple vascular risk factors [55]. Collaboratively, our findings are consistent with those of others that argued that a decline in SLF white matter integrity has associations with uncontrolled vascular risk factors [56,57]. Changes in diffusion parameters in SLF have also been found to be associated with increased QRISK2; for instance, increased RD in the SLF has also been reported in subjects with dyslipidemia [57], without any report on bilateral involvements. Hence, our findings may infer that changes in the integrity of SLF tracts regarding cardiocerebrovascular risk appear to be more dominant in the left hemisphere (i.e., LSLF). In addition, the QRISK2 percentage score may serve as a practical parallel tool to gauge the integrity of SLF white matter tracts-at least, in the setting of asymptomatic subjects, for which occult CSVD may incidentally be detected in neuroimaging.

Furthermore, no significant relationship was found between age and FA, although the results suggested reduced SLF white matter integrity in older subjects. Hence, we postulate that advancing age may only negatively impact a portion of SLF. For instance, a previous study has shown that aging selectively impacts frontal white matter regions [58-60]. Several DTI studies have supported this "frontal aging hypothesis" and showed an anterior to posterior gradient in the decline of white matter integrity, with reduced FA and increased MD of frontal lobe structures despite the relative preservation of posterior brain regions [61-65].

In a routine clinical setting, our study supports the notion that an increased cardiocerebrovascular risk (as predicted by QRISK2 score) may serve as an objective and diagnostic guide to inform clinicians about the potential risk of underlying WMHs, specifically with respect to the CSVD spectrum in asymptomatic, economically productive young adults.

\subsection{Neurocognitive Profiles as Surrogate Markers to CSVD}

It has been reported that $\mathrm{WMH}^{+}$subjects with vascular risk factors are more vulnerable to developing cognitive impairment [66]. Throughout the present study, only PSI was consistently correlated and associated with age and not with PRI and WMI. The variability of results between WAIS-IV indices used in the present study may be due to the small sample size and lack of follow-up. In addition, we recruited asymptomatic subjects as young as 25 years old. One study reported that, among 142 subjects, those aged 20-59 years $(n=95)$ had WMHs burden, which was not significantly associated with cognitive deficits, whereas participants aged 60 and older $(n=47)$ accounted for more than $58 \%$ of the variance in working memory, processing speed, fluency, and fluid intelligence performances [67]. 
The Ohasama Study [68] reported among a population of healthy Japanese adults ( $n=331$, age $\geq 60$ years) with a 7 year follow-up that reductions in processing speed, perceptual reasoning, and memory appeared to be associated with silent cerebrovascular white matter lesions or WMHs. Similar findings have also been reported with respect to the association of the presence of WMHs with frontal executive function, memory, and global functioning [69]. By contrast, Sachdev et al. (2017) [70] did not find any significant association between the prevalence of WMHs with cognitive functions, especially on episodic memory, working memory, and general intellectual functioning in 478 subjects aged 60-64 years.

Furthermore, in the present study, we found a significant relationship with processing speed on the basis of PSI and age (but not QRISK2), where PSI was lower in $\mathrm{WMH}^{+}$subjects. This finding was supported by the fact that the progression of WMHs in periventricular spaces has been recognized to affect cognitive impairment, particularly in terms of information processing speed and long-term functional decline [71]. However, both studies involved a population-based sample of non-demented and stroke-free individuals ranging between 60 and 90 years old, with a bigger sample size (sample size up to 1000). Finally, WMHs have also been previously reported to predict cognitive impairments in the elderly (age $\geq 60$ years) with depression but were not associated with cognitive performance in healthy controls [72]. The total impact of WMHs burden has been reported to be minimal and unrelated to reduced cognitive performance among young-to-middle-aged adults (20-59 years old); however, WMHs have been frequently associated with low cognitive test performance among adults age 60 years and older [73].

We found no association between neurocognitive profiles and diffusion parameters, although a significant correlation was found between the profiles with FA of selected white matter tracts. According to Tuladhar et al., reduced white matter tract integrity in certain locations of white matter increased the likelihood of decline in specific cognitive functions [74]. Similarly, D'Souza et al. showed that changes of FA in white matter tracts significantly affected the performance of subjects in terms of cognitive function tests [75]. The Radboud University Nijmegen Diffusion Tensor and Magnetic Resonance Cohort (RUN DMC) cohort [76] initially studied 499 subjects with a prevalence of WMHs using WAIS digit span evaluations and found that FA was associated with attention and processing speed. The subsequent work of this cohort further demonstrated reduced FA and higher MD in the white matter tracts of symptomatic CSVD subjects $(n=115)$ compared with asymptomatic controls $(n=50)$, insinuating an association of white matter integrity with processing speed and executive function [77]. A similar observation has been reported in the Discontinuation of Antihypertensive Treatment in Elderly People (DANTE) study in Leiden [78], regarding cognitive and memory functions in healthy adults. Thus, the findings in the present study support the growing evidence indicating that white matter integrity, in terms of FA, can potentially be used as a neuroimaging marker for cognitive and memory ability in asymptomatic individuals, with or without the evidence of WMHs.

\subsection{Microparticles as Surrogate Markers for CSVD}

To the best of our knowledge, this research is the first to describe and show an elevated MPs counts (mean percentage) in asymptomatic $\mathrm{WMH}^{+}$subjects, concerning select MP subpopulations. We found that platelets-derived MPs (PDMPs) (CD62P), leukocyte-derived MPs (LMPs) (CD62L), and total MPs count were significantly higher among $\mathrm{WMH}^{+}$subjects. This may suggest the potential roles of certain MPs subpopulations (i.e., PDMPs and LMPs) in the prevalence of WMHs among our asymptomatic healthy subjects as well as the contribution of different MPs subpopulations to the total MPs count.

However, there is a lack in the literature regarding changes associated with the level of circulating MPs in asymptomatic individuals. To date, most studies have compared differences in the levels of MPs between healthy controls and symptomatic patients in vascular-related diseases. A higher level of LMPs in ischemic cerebrovascular disease patients compared with normal controls has been reported [79]. In addition, an increment 
in the level of endothelial cell-derived MPs (EMPs) (CD146) has also been found in CSVD symptomatic patients compared with healthy controls [15], thus depicting that EMPs may contribute to the progression of CSVD. Furthermore, several studies have found increases in the level of CD62P in symptomatic, lacunar stroke patients [78,80] only. Thus, elaborating the role of MPs levels in asymptomatic individuals may contribute to predicting future symptomatic events, if the MPs can be affirmatively determined as surrogate blood biomarkers for the spectrum of CSVD manifestations, including "silent" WMHs.

PDMPs are a major contributor to microthrombi [81], supporting the findings of the present study, as PDMPs had the highest counts in $\mathrm{WMH}^{+}$subjects. A cross-sectional study among 190 healthy Japanese men with predicted 10-year cardiocerebrovascular risk found that higher PDMPs were associated with increased cardiovascular diseases [82]. In another study, PDMPs were assumed to play a significant role in cardiovascular disease, serving as a major player in coagulation and the formation of microthrombi in blood vessels [83]. PDMPs have also been significantly associated with lesion volume in acute ischemic stroke but not in asymptomatic subjects with WMHs lesions on neuroimaging [84]. It is of interest that we found only LMPs (CD62L) to be associated with 10-year cardiocerebrovascular risk (QRISK2), rather than PDMPs, and to also not be associated with age and QRISK2 (see Figure 2).

Higher circulating LMPs have been demonstrated in patients with ischemic cerebrovascular disease [79], where healthy controls had a relatively low count compared with those who were symptomatic. As described in previous studies, greater counts of LMPs have been found in patients with recent cardiovascular events, thus inferring that an elevated level of circulating MPs reflects the underlying vessel occlusion in the form of microthrombi in symptomatic patients $[85,86]$. An increased risk of thrombosis has also been reported, concerning elevated LMPs levels in cardiovascular risk patients, when compared with healthy controls [87]. Thus, collectively, it seems that increased circulating MPs subpopulations (i.e., PDMPs and/or LMPs) and combinations of multiple MPs subpopulations (i.e., total MPs) have a potential predictive impact on the prevalence and progression of WMHs in CSVD among asymptomatic individuals (and, in the present study, among those aged 25-62 years; see Figure 2). Further research is required to confirm these findings, preferably with a follow-up study and collective population data.

In the present study, circulating MPs were featured as novel surrogate markers for the white matter integrity in CVSD, through the presence/absence of WMHs. However, no significant correlation was found between MPs and the diffusion parameters of the selected tracts. Nevertheless, significant associations were found between LSLF diffusion parameters and CD62L, CD146, and total MPs. A plausible reason for this attracts an argument based on a previous study, which opined that increased FA in certain tracts might also be due to decreases in axonal diameter and branching [36]. Essentially, it is presumed that the occluded small penetrating vessels inflict a subclinical ischemic insult in the LSLF region, which may potentially contribute to the decline in white matter integrity in that specific tract over time unless perfusion is restored. However, the exact molecular and cellular mechanisms underlying the changes in diffusion parameters (i.e., FA) remain unknown [88]. Hence, careful interpretation on the changes in FA is necessary, as increased FA can also be due to neuronal degeneration, such as in crossing fibers [89].

Furthermore, changes in the AD parameter of the LSLF were found to correlate with higher circulating CD62L, CD146, and total MPs count. Previous studies (in both humans and animal) have indicated that the reduction of AD might be due to the effects of ischemia, thus suggesting that axonal degeneration (regarding ischemia) may lead to a subsequent reduction in diffusion coherence in terms of directionality (i.e., AD) [90,91]. These, too, appear to arise from aging, by which (as described before) AD seems to decrease with age, chiefly from age-related demyelination [92]. It is of interest that, in the present study, we also found that CD62L is associated with QRISK2 and the PRI cognitive index, whereas total MPs were exclusively associated with PRI performance. Hence, it can be speculated that the CD62L subpopulation within the micro-thrombus formation may play a crucial 
role in inducing ischemic demyelination in CSVD (see Figure 2) and, thus, may serve as a plausible biomarker for vascular-related cognitive impairment associated with CSVD.

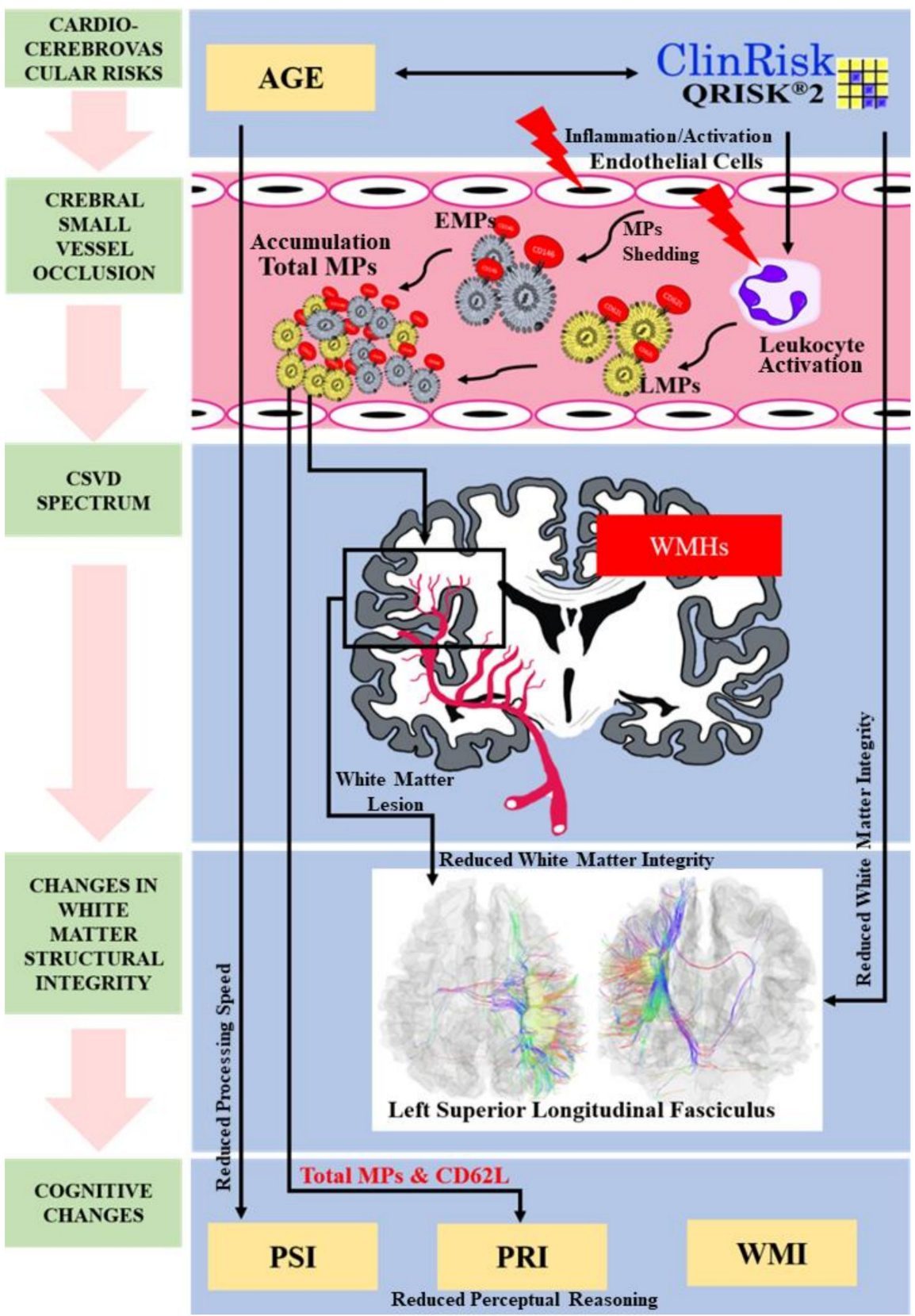

Figure 2. Multimodal linear relationship between surrogate markers: cardiocerebrovascular risk factors, such as age, are associated with QRISK2 and Wechsler Adult Intelligence Scale: Fourth edition (WAIS-IV) processing speed index (PSI). QRISK2 is associated with the leukocyte-derived MPs (LMPs; i.e., CD62L), whereas endothelial cell-derived MPs (EMPs; i.e., CD146) may exclusively be induced by other factors, inclusive of cardiocerebrovascular risk factors. LMPs and EMPs can potentially contribute to total MPs; hence, MP accumulation in cerebral small vessels (or end arteries) is thus postulated to cause occlusion. Such an occlusion may induce the formation of WMHs. LMPs, EMPs, and total MPs were associated with the integrity of the left superior longitudinal fasciculus (LSLF) regarding the presence of WMHs in that particular region. QRISK2 was also directly associated with the integrity of LSLF. Finally, total MPs and CD62L were exclusively associated with the WAIS-IV perceptual reasoning index (PRI). 


\subsection{Strengths and Limitations of the Study}

In this study, we established multimodal associations as potential surrogate markers for "silent" CSVD manifestation in well-characterized cardiocerebrovascular demographics among a relatively young sample of neurologically asymptomatic adults. To the best of our knowledge, this study is the first to exhibit elevated MPs counts in asymptomatic CSVD, which warrants further delineation. However, the study was conducted in a single center clinical setting with small sample size $(n=60)$ in order to explore the prevalence of WMHs, thus limiting the generalizability of the study findings. Moreover, as part of the study inclusion/exclusion criteria, many older adults were excluded due to their high QRISK2 scores (more than 20\%) and were regarded as symptomatic individuals. In addition, WAIS-IV is labor-intensive and client-demanding to complete, which better suits the research setting and is impractical for routine daily clinical use for neurocognitive screening. Nevertheless, we consolidated the relevant WAIS-IV indices for the purposes of this research in order to ensure comprehensiveness of the assessment. Finally, the candidate MPs selected and tested in this study represent a panel of MPs, selected among other MPs reported at the time of research commencement. Thus, extending the involvement of other/new MPs subpopulations in this context remains unknown.

\section{Conclusions}

The present study, to the best of our knowledge, was the first to examine the presence/absence of WMHs to depict CSVD in apparently asymptomatic individuals using three modalities, namely, neurocognitive, microparticles profiling, and neuroimaging (dMRI), in a selected cohort of subjects with variable cardiocerebrovascular risk (as determined by QRISK2 predictive score). Although it was carried out at a single center with a small sample size, the present study established "silent" CSVD manifestations in apparently asymptomatic individuals from well-characterized cardiovascular demographics in relatively young, neurologically asymptomatic adults from suburban southeast Peninsular Malaysia.

Author Contributions: Conceptualization, C.M.N.C.M.N. and M.M.; methodology, C.M.N.C.M.N., M.M.G., A.A.S., and M.M.; software, C.M.N.C.M.N. and U.J.; validation, W.Z.A., N.S.I., and M.M.; formal analysis, C.M.N.C.M.N.; investigation, C.M.N.C.M.N. and U.J.; resources, C.M.N.C.M.N and M.M.G.; data curation, C.M.N.C.M.N.; Writing-Original draft preparation, C.M.N.C.M.N.; Writing-Review and editing, C.M.N.C.M.N.; visualization, C.M.N.C.M.N.; supervision, M.M.; project administration, C.M.N.C.M.N. and M.M.; funding acquisition, M.M. All authors have read and agreed to the published version of the manuscript.

Funding: This research was funded by Fundamental Research Grant Scheme (FRGS), Ministry of Higher Education Malaysia, grant number 203/PPSP/61771193.

Institutional Review Board Statement: The study was conducted according to the guidelines of the Declaration of Helsinki and approved by the Human Research Ethics Committee at Universiti Sains Malaysia (Protocol code: USM/JEPeM/15030096).

Informed Consent Statement: Informed consent was obtained from all subjects involved in the study.

Data Availability Statement: We declare that the materials described in the manuscript, including all relevant raw data, will be freely available to any scientist wishing to use them for noncommercial purposes, without breaching participant confidentiality.

Acknowledgments: We thank the management of the Hospital Universiti Sains Malaysia for granting permission to the investigators to use patient medical records as well as space and assets belonging to the hospital during conduction of the research.

Conflicts of Interest: The authors declare no potential conflict of interest with respect to the research, authorship, and/or publication of this article. 


\section{References}

1. Mustapha, M.; Nassir, C.M.N.C.M.; Aminuddin, N.; Safri, A.A.; Ghazali, M.M. Cerebral small vessel disease (CSVD)—Lessons from the animal models. Front. Physiol. 2019, 10, 1317. [CrossRef] [PubMed]

2. Lammie, A.G. Small vessel disease. In Cerebrovascular Diseases; Kalimo, H., Ed.; ISN Neuropath Press: Basel, Switzerland, 2005; pp. 85-91.

3. Pantoni, L. Cerebral small vessel disease: From pathogenesis and clinical characteristics to therapeutic challenges. Lancet Neurol. 2010, 9, 689-701. [CrossRef]

4. Ogata, J.; Yamanishi, H.; Ishibashi-Ueda, H. Pathology of cerebral small vessel disease. In Cerebral Small Vessel Disease; Pantoni, L., Gorelick, P.B., Eds.; Cambridge University Press: Cambridge, UK, 2014; pp. 4-15.

5. Hachinski, V. World stroke Day 2008: "Little strokes, big trouble". Stroke 2008, 39, 2407-2420. [CrossRef] [PubMed]

6. Thompson, C.S.; Hakim, A.M. Living beyond our physiological means: Small vessel disease of the brain is an expression of a systemic failure in arteriolar function: A unifying hypothesis. Stroke 2009, 40, e322-e330. [CrossRef]

7. Gunda, B.; Bereczki, D.; Várallyay, G. Multimodal MRI of cerebral small vessel disease. In Neuroimaging-Clinical Applications; Bright, P., Ed.; INTECH Open Access Publisher: London, UK, 2012; pp. 277-291.

8. Das, A.S.; Regenhardt, R.W.; Vernooij, M.W.; Blacker, D.; Charidimou, A.; Viswanathan, A. Asymptomatic cerebral small vessel disease: Insights from population-based studies. J. Stroke 2019, 21, 121-138. [CrossRef]

9. Smith, E.E. Leukoaraiosis and stroke. Stroke 2010, 41, S139-S143. [CrossRef]

10. Patel, B.; Markus, H.S. Magnetic resonance imaging in cerebral small vessel disease and its use as a surrogate disease marker. Int. J. Stroke 2011, 6, 47-49. [CrossRef]

11. Rost, N.S.; Rahman, R.M.; Biffi, A.; Smith, E.E.; Kanakis, A.; Fitzpatrick, K.; Lima, F.; Worrall, B.B.; Meschia, J.F.; Brown, R.D.; et al. White matter hyperintensity volume is increased in small vessel stroke subtypes. Neurology 2010, 75, 1670-1677. [CrossRef]

12. Huynh, T.J.; Murphy, B.; Pettersen, J.A.; Tu, H.; Sahlas, D.J.; Zhang, L.; Symons, S.P.; Black, S.; Lee, T.Y.; Aviv, R.I. CT perfusion quantification of small-vessel ischemic severity. AJNR. Am. J. Neuroradiol. 2008, 29, 1831-1836. [CrossRef]

13. Kanjilal, S.; Rao, V.S.; Mukherjee, M.; Natesha, B.K.; Renuka, K.S.; Sibi, K.; Iyengar, S.S.; Kakkar, V.V. Application of cardiovascular disease risk prediction models and the relevance of novel biomarkers to risk stratification in Asian Indians. Vasc. Health Risk Manag. 2008, 4, 199-211. [CrossRef]

14. Collins, G.S.; Altman, D.G. Predicting the 10-year risk of cardiovascular disease in the United Kingdom: Independent and external validation of an updated version of QRISK2. BMJ 2012, 344, e4181. [CrossRef] [PubMed]

15. Berezin, A.E.E. Impaired phenotype of circulating endothelial-derived microparticles: Novel marker of cardiovascular risk. J. Cardiol. Ther. 2015, 2, 365-370. [CrossRef]

16. Dignat-George, F.; Boulanger, C.M. The many faces of endothelial microparticles. Arterioscler. Thromb. Vasc. Biol. 2011, 31, 27-33. [CrossRef]

17. Bebawy, M.; Roseblade, A.; Luk, F.; Rawling, T.; Ung, A.; Grau, G.E.R. Cell-derived microparticles: New targets in the therapeutic management of disease. J. Pharm. Pharm. Sci. 2013, 16, 238. [CrossRef] [PubMed]

18. Chiva-Blanch, G.; Suades, R.; Crespo, J.; Vilahur, G.; Arderiu, G.; Padró, T.; Corella, D.; Salas-Salvadó, J.; Arós, F.; Martínez-González, M.A. CD3/CD45 and SMA- $\alpha$ circulating microparticles are increased in individuals at high cardiovascular risk who will develop a major cardiovascular event. Int. J. Cardiol. 2016, 208, 147-149. [CrossRef]

19. Nomura, S.; Shimizu, M. Clinical significance of procoagulant microparticles. J. Intensiv. Care 2015, 3, 2. [CrossRef]

20. Owens, A.P.; Mackman, N. Microparticles in hemostasis and thrombosis. Circ. Res. 2011, 108, 1284-1297. [CrossRef]

21. Andriantsitohaina, R.; Gaceb, A.; Vergori, L.; Martínez, M.C. Microparticles as regulators of cardiovascular inflammation. Trends Cardiovasc. Med. 2012, 22, 88-92. [CrossRef]

22. Martinez, M.C.; Tual-Chalot, S.; Leonetti, D.; Andriantsitohaina, R. Microparticles: Targets and tools in cardiovascular disease. Trends Pharmacol. Sci. 2011, 32, 659-665. [CrossRef]

23. Burger, D.; Touyz, R.M. Cellular biomarkers of endothelial health: Microparticles, endothelial progenitor cells, and circulating endothelial cells. J. Am. Soc. Hypertens. 2012, 6, 85-89. [CrossRef]

24. Grammas, P.; Martinez, J.; Miller, B. Cerebral microvascular endothelium and the pathogenesis of neurodegenerative diseases. Expert Rev. Mol. Med. 2011, 13, e19. [CrossRef] [PubMed]

25. Ogata, J.; Yamanishi, H.; Ishibashi-Ueda, H. Review: Role of cerebral vessels in ischaemic injury of the brain. Neuropathol. Appl. Neurobiol. 2011, 37, 40-45. [CrossRef] [PubMed]

26. Schreiber, S.; Bueche, C.Z.; Garz, C.; Braun, H. Blood brain barrier breakdown as the starting point of cerebral small vessel disease?-New insights from a rat model. Exp. Transl. Stroke Med. 2013, 5, 4. [CrossRef] [PubMed]

27. Poncelet, P.; Robert, S.; Bailly, N.; Garnache-Ottou, F.; Bouriche, T.; Devalet, B.; Segatchian, J.H.; Saas, P.; Mullier, F. Tips and tricks for flow cytometry-based analysis and counting of microparticles. Transfus. Apher. Sci. 2015, 53, 110-126. [CrossRef]

28. Lacroix, R.; Judicone, C.; Poncelet, P.; Robert, S.; Arnaud, L.; Sampol, J.; Dignat-George, F. Impact of pre-analytical parameters on the measurement of circulating microparticles: Towards standardization of protocol. J. Thromb. Haemost. 2012, 10, 437-446. [CrossRef]

29. Mooberry, M.J.; Bradford, R.; Hobl, E.L.; Lin, F.C.; Jilma, B.; Key, N.S. Procoagulant microparticles promote coagulation in a factor XI-dependent manner in human endotoxemia. J. Thromb. Haemost. 2016, 14, 1031-1042. [CrossRef] 
30. Nielsen, M.H.; Beck-Nielsen, H.; Andersen, M.N.; Handberg, A. A flow cytometric method for characterization of circulating cell-derived microparticles in plasma. J. Extracell. Vesicles 2014, 3, 20795. [CrossRef]

31. Chiva-Blanch, G.; Suades, R.; Crespo, J.; Peña, E.; Padró, T.; Jiménez-Xarrié, E.; Martí-Fàbregas, J.; Badimon, L. Microparticle Shedding from Neural Progenitor Cells and Vascular Compartment Cells Is Increased in Ischemic Stroke. PLoS ONE 2016, 11, e0148176. [CrossRef]

32. Toussaint, N.; Souplet, J.C.; Fillard, P. MedINRIA: Medical image navigation and research tool by INRIA. In Proceedings of the MICCAI'07 Workshop on Interaction in Medical Image Analysis and Visualization, Brisbane, Australia, 29 October-2 November 2007. (inria-00616047).

33. Fazekas, F.; Chawluk, J.B.; Alavi, A.; Hurtig, H.I.; Zimmerman, R.A. MR signal abnormalities at 1.5 T in Alzheimers dementia and normal aging. Am. J. Roentgenol. 1987, 149, 351-356. [CrossRef]

34. Griffanti, L.; Jenkinson, M.; Suri, S.; Zsoldos, E.; Mahmood, A.; Filippini, N.; Sexton, C.E.; Topiwala, A.; Allan, C.; Kivimäki, M.; et al. Classification and characterization of periventricular and deep white matter hyperintensities on MRI: A study in older adults. Neuroimage 2018, 170, 174-181. [CrossRef]

35. Yeh, F.C.; Verstynen, T.D.; Wang, Y.; Fernández-Miranda, J.C.; Tseng, W.Y. Deterministic diffusion fiber tracking improved by quantitative anisotropy. PLoS ONE 2013, 8, e80713. [CrossRef] [PubMed]

36. Beaulieu, C. The basis of anisotropic water diffusion in the nervous system-A technical review. NMR Biomed. 2002, 15, 435-455. [CrossRef] [PubMed]

37. Pierpaoli, C.; Jezzard, P.; Basser, P.J.; Barnett, A.; Di Chiro, G.D. Diffusion tensor MR imaging of the human brain. Radiology 1996, 201, 637-648. [CrossRef] [PubMed]

38. Basser, P.J. Inferring microstructural features and the physiological state of tissues from diffusion-weighted images. NMR Biomed. 1995, 8, 333-344. [CrossRef] [PubMed]

39. Bennett, I.J.; Madden, D.J.; Vaidya, C.J.; Howard, D.V.; Howard, J.H. Age-related differences in multiple measures of white matter integrity: A diffusion tensor imaging study of healthy aging. Hum. Brain Mapp. 2010, 31, 378-390. [CrossRef]

40. Breteler, M.; Van Swieten, J.C.; Bots, M.L.; Grobbee, D.E.; Claus, J.J.; Hout, J.H.V.D.; Van Harskamp, F.; Tanghe, H.L.; De Jong, P.T.; Van Gijn, J.; et al. Cerebral white matter lesions, vascular risk factors, and cognitive function in a population-based study: The Rotterdam Study. Neurology 1994, 44, 1246. [CrossRef]

41. Raz, N.; Yang, Y.; Dahle, C.L.; Land, S. Volume of white matter hyperintensities in healthy adults: Contribution of age, vascular risk factors, and inflammation-related genetic variants. Biochim. Biophys. Acta 2012, 1822, 361-369. [CrossRef]

42. Sam, K.; Peltenburg, B.; Conklin, J.; Sobczyk, O.; Poublanc, J.; Crawley, A.P.; Mandell, D.M.; Venkatraghavan, L.; Duffin, J.; Fisher, J.A.; et al. Cerebrovascular reactivity and white matter integrity. Neurology 2016, 87, 2333-2339. [CrossRef]

43. Qiu, W.Q.; Himali, J.J.; Wolf, P.A.; Decarli, D.C.; Beiser, A.; Au, R. Effects of white matter integrity and brain volumes on late life depression in the Framingham Heart Study. Int. J. Geriatr. Psychiatry 2017, 32, 214-221. [CrossRef]

44. Kilpatrick, E.P.; Buckley, R.F.; Marshall, G.A.; Klein, H.; Properzi, M.; Schultz, A.P.; Rao, V.; Rabin, J.S.; Hanseeuw, B.; Rentz, D.M.; et al. [P3-376]: Qrisk2 and framingham cardiovascular risk scores significantly correlate with imaging biomarkers of preclinical Ad: Findings from the harvard aging brain study. Alzheimers Dement. 2017, 13, P1103. [CrossRef]

45. Armstrong, A.C.; Muller, M.; Ambale-Ventakesh, B.; Halstead, M.; Kishi, S.; Bryan, N.; Sidney, S.; Correia, L.C.; Gidding, S.S.; Launer, L.J. Association of early left ventricular dysfunction with advanced magnetic resonance white matter and gray matter brain measures: The CARDIA study. Echocardiography 2017, 34, 1617-1622. [CrossRef] [PubMed]

46. Hilal, S.; Mok, V.; Youn, Y.C.; Wong, A.; Ikram, M.K.; Chen, C.L.-H. Prevalence, risk factors and consequences of cerebral small vessel diseases: Data from three Asian countries. J. Neurol. Neurosurg. Psychiatry 2017, 88, 669-674. [CrossRef] [PubMed]

47. Hilal, S.; Tan, C.S.; Xin, X.; Amin, S.M.; Wong, T.Y.; Chen, C.; Venketasubramanian, N.; Ikram, M.K. Prevalence of Cognitive Impairment and Dementia in Malays - Epidemiology of Dementia in Singapore Study. Curr. Alzheimer Res. 2017, 14, 620-627. [CrossRef] [PubMed]

48. Nyquist, P.A.; Bilgel, M.S.; Gottesman, R.; Yanek, L.R.; Moy, T.F.; Becker, L.C.; Cuzzocreo, J.; Prince, J.; Yousem, D.M.; Becker, D.M. Extreme deep white matter hyperintensity volumes are associated with African American race. Cerebrovasc. Dis. 2014, 37, 244-250. [CrossRef]

49. Mok, V.C.T.; Srikanth, V.K.; Xiong, Y.; Phan, T.G.; Moran, C.; Chu, S.; Zhao, Q.; Chu, W.C.-W.; Wong, A.; Hong, Z.; et al. RaceEthnicity and Cerebral Small Vessel Disease - Comparison between Chinese and White Populations. Int. J. Stroke 2014, 9, 36-42. [CrossRef]

50. Sedaghat, S.; Cremers, L.G.; De Groot, M.; Hofman, A.; Van Der Lugt, A.; Niessen, W.; Franco, O.H.; Dehghan, A.; Ikram, M.K.; Vernooij, M.W. Lower microstructural integrity of brain white matter is related to higher mortality. Neurology 2016, 87, 927-934. [CrossRef]

51. Gons, R.A.; Tuladhar, A.M.; de Laat, K.F.; van Norden, A.G.; van Dijk, E.J.; Norris, D.G.; Zwiers, M.P.; de Leeuw, F.E. Physical activity is related to the structural integrity of cerebral white matter. Neurology 2013, 81, 971-976. [CrossRef]

52. Gons, R.A.; van Norden, A.G.; de Laat, K.F.; van Oudheusden, L.J.; van Uden, I.W.; Zwiers, M.P.; Norris, D.G.; de Leeuw, F.E. Cigarette smoking is associated with reduced microstructural integrity of cerebral white matter. Brain 2011, 134, $2116-2124$. [CrossRef]

53. Gons, R.A.; de Laat, K.F.; van Norden, A.G.; van Oudheusden, L.J.; van Uden, I.W.; Norris, D.G.; Zwiers, M.P.; de Leeuw, F.E. Hypertension and cerebral diffusion tensor imaging in small vessel disease. Stroke 2010, 41, 2801-2806. [CrossRef] 
54. Lyoubi-Idrissi, A.L.; Jouvent, E.; Poupon, C.; Chabriat, H. Diffusion magnetic resonance imaging in cerebral small vessel disease. Rev. Neurol. 2017, 173, 201-210. [CrossRef]

55. Haight, T.; Bryan, R.N.; Erus, G.; Hsieh, M.-K.; Davatzikos, C.; Nasrallah, I.; D’Esposito, M.; Jacobs, D.R.; Lewis, C.; Schreiner, P.; et al. White matter microstructure, white matter lesions, and hypertension: An examination of early surrogate markers of vascular-related brain change in midlife. Neuroimage Clin. 2018, 18, 753-761. [CrossRef] [PubMed]

56. Alfaro, F.J.; Gavrieli, A.; Saade-Lemus, P.; Lioutas, V.A.; Upadhyay, J.; Novak, V. White matter microstructure and cognitive decline in metabolic syndrome: A review of diffusion tensor imaging. Metab. Clin. Exp. 2018, 78, 52-58. [CrossRef] [PubMed]

57. Williams, V.J.; Leritz, E.C.; Shepel, J.; McGlinchey, R.E.; Milberg, W.P.; Rudolph, J.L.; Lipsitz, L.A.; Salat, D.H. Interindividual variation in serum cholesterol is associated with regional white matter tissue integrity in older adults. Hum. Brain Mapp. 2013, 34, 1826-1841. [CrossRef] [PubMed]

58. Dempster, F.N. The rise and fall of the inhibitory mechanism: Toward a unified theory of cognitive development and aging. Dev. Rev. 1992, 12, 45-55. [CrossRef]

59. Greenwood, P.M. The frontal aging hypothesis evaluated. J. Int. Neuropsychol. Soc. 2000, 6, 705-726. [CrossRef]

60. West, R.L. An application of prefrontal cortex function theory to cognitive aging. Psychol. Bull. 1996, 120, 272-292. [CrossRef]

61. Abe, O.; Yamasue, H.; Aoki, S.; Suga, M.; Yamada, H.; Kasai, K.; Masutani, Y.; Kato, N.; Kato, N.; Ohtomo, K. Aging in the CNS: Comparison of gray/white matter volume and diffusion tensor data. Neurobiol. Aging 2008, 29, 102-116. [CrossRef]

62. Lehmbeck, J.T.; Brassen, S.; Weber-Fahr, W.; Braus, D.F. Combining voxel-based morphometry and diffusion tensor imaging to detect age-related brain changes. Neuroreport 2006, 17, 467-470. [CrossRef]

63. Pfefferbaum, A.; Adalsteinsson, E.; Sullivan, E.V. Frontal circuitry degradation marks healthy adult aging: Evidence from diffusion tensor imaging. Neuroimage 2005, 26, 891-899. [CrossRef]

64. Salat, D.H.; Tuch, D.; Greve, D.; Van Der Kouwe, A.; Hevelone, N.; Zaleta, A.; Rosen, B.; Fischl, B.; Corkin, S.; Rosas, H.D.; et al Age-related alterations in white matter microstructure measured by diffusion tensor imaging. Neurobiol. Aging 2005, 26, 1215-1227. [CrossRef]

65. Stamatakis, E.A.; Shafto, M.A.; Williams, G.; Tam, P.; Tyler, L.K. White matter changes and word finding failures with increasing age. PLoS ONE 2011, 6, e14496. [CrossRef] [PubMed]

66. Kynast, J.; Lampe, L.; Luck, T.; Frisch, S.; Arelin, K.; Hoffmann, K.-T.; Loeffler, M.; Riedel-Heller, S.G.; Villringer, A.; Schroeter, M. White matter hyperintensities associated with small vessel disease impair social cognition beside attention and memory. Br. J. Pharmacol. 2018, 38, 996-1009. [CrossRef] [PubMed]

67. Chen, T.; Li, D. The roles of working memory updating and processing speed in mediating age-related differences in fluid intelligence. Neuropsychology, Development, and Cognition. Sect. B Aging Neuropsychol. Cogn. 2007, 14, 631-646. [CrossRef] [PubMed]

68. Tsubota-Utsugi, M.; Satoh, M.; Tomita, N.; Hara, A.; Kondo, T.; Hosaka, M.; Saito, S.; Asayama, K.; Inoue, R.; Hirano, M.; et al. Lacunar Infarcts Rather than White Matter Hyperintensity as a Predictor of Future Higher Level Functional Decline: The Ohasama Study. J. Stroke Cerebrovasc. Dis. 2017, 26, 376-384. [CrossRef] [PubMed]

69. De Groot, M.D.; Anderson, R.; Freedland, K.E.; Clouse, R.E.; Lustman, P.J. Association of Depression and Diabetes Complications: A meta-analysis. Psychosom. Med. 2001, 63, 619-630. [CrossRef]

70. Sachdev, P.S.; Lo, J.W.; Crawford, J.D.; Mellon, L.; Hickey, A.; Williams, D.; Bordet, R.; Mendyk, A.M.; Gelé, P.; Deplanque, D STROKOG (stroke and cognition consortium): An international consortium to examine the epidemiology, diagnosis, and treatment of neurocognitive disorders in relation to cerebrovascular disease. Alzheimers Dement. 2017, 7, 11-13. [CrossRef]

71. Dhamoon, M.S.; Cheung, Y.K.; Bagci, A.; Alperin, N.; Sacco, R.L.; Elkind, M.S.V.; Wright, C.B. Periventricular white matter hyperintensities and functional decline. J. Am. Geriatr. Soc. 2018, 66, 113-119. [CrossRef]

72. Köhler, S.; Thomas, A.J.; Lloyd, A.; Barber, R.; Almeida, O.P.; O’Brien, J.T. White matter hyperintensities, cortisol levels, brain atrophy and continuing cognitive deficits in late-life depression. Br. J. Psychiatry 2010, 196, 143-149. [CrossRef]

73. Vannorsdall, T.D.; Waldstein, S.R.; Kraut, M.; Pearlson, G.D.; Schretlen, D.J. White matter abnormalities and cognition in a community sample. Arch. Clin. Neuropsychol. 2009, 24, 209-217. [CrossRef]

74. Tuladhar, A.M.; van Norden, A.G.; de Laat, K.F.; Zwiers, M.P.; van Dijk, E.J.; Norris, D.G.; de Leeuw, F.E. White matter integrity in small vessel disease is related to cognition. Neuroimage Clin. 2015, 7, 518-624. [CrossRef]

75. D'Souza, M.M.; Gorthi, S.P.; Vadwala, K.; Trivedi, R.; Vijayakumar, C.; Kaur, P.; Khushu, S. Diffusion tensor tractography in cerebral small vessel disease: Correlation with cognitive function. Neuroradiol. J. 2018, 31, 83-89. [CrossRef] [PubMed]

76. Van Norden, A.G.; de Laat, K.F.; Gons, R.A.; van Uden, I.W.; van Dijk, E.J.; van Oudheusden, L.J.; Esselink, R.A.; Bloem, B.R.; van Engelen, B.G.; Zwarts, M.J. Causes and consequences of cerebral small vessel disease. The RUN DMC study: A prospective cohort study. Study rationale and protocol. BMC Neurol. 2011, 11, 29. [CrossRef] [PubMed]

77. Lawrence, A.J.; Patel, B.; Morris, R.G.; MacKinnon, A.D.; Rich, P.M.; Barrick, T.R.; Markus, H.S. Mechanisms of cognitive impairment in cerebral small vessel disease: Multimodal MRI results from the St George's cognition and neuroimaging in stroke (SCANS) study. PLoS ONE 2013, 8, e61014. [CrossRef]

78. Moonen, J.E.F.; Foster-Dingley, J.C.; Berg-Huijsmans, A.A.V.D.; De Ruijter, W.; De Craen, A.J.M.; Van Der Grond, J.; Van Der Mast, R. Influence of Small Vessel Disease and Microstructural Integrity on Neurocognitive Functioning in Older Individuals: The DANTE Study Leiden. Am. J. Neuroradiol. 2016, 38, 25-30. [CrossRef] [PubMed] 
79. He, Z.; Tang, Y.; Qin, C. Increased circulating leukocyte-derived microparticles in ischemic cerebrovascular disease. Thromb. Res. 2017, 154, 19-25. [CrossRef] [PubMed]

80. Wiseman, S.; Marlborough, F.; Doubal, F.; Webb, D.J.; Wardlaw, J. Blood Markers of Coagulation, Fibrinolysis, Endothelial Dysfunction and Inflammation in lacunar Stroke versus Non-Lacunar Stroke and Non-Stroke: Systematic Review and MetaAnalysis. Cerebrovasc. Dis. 2014, 37, 64-65. [CrossRef] [PubMed]

81. Suades, R.; Padró, T.; Vilahur, G.; Badimon, L. C0074 Increased number of circulating and platelet-derived microparticles in human blood enhances thrombosis on atherosclerotic plaques. Thromb. Res. 2012, 130, S115. [CrossRef]

82. Ueba, T.; Nomura, S.; Inami, N.; Nishikawa, T.; Kajiwara, M.; Iwata, R.; Yamashita, K. Plasma level of platelet-derived microparticles is associated with coronary heart disease risk score in healthy men. J. Atheroscler. Thromb. 2010, 17, 342-349. [CrossRef]

83. Viera, A.J.; Mooberry, M.; Key, N.S. Microparticles in cardiovascular disease pathophysiology and outcomes. J. Am. Soc. Hypertens. 2012, 6, 243-252. [CrossRef]

84. Simak, J.; Gelderman, M.P.; Yu, H.; Wright, V.; Baird, A.E. Circulating endothelial microparticles in acute ischemic stroke: A link to severity, lesion volume and outcome. J. Thromb. Haemost. 2006, 4, 1296-1302. [CrossRef]

85. Suades, R.; Padro, T.; Crespo, J.; Ramaiola, I.; Martin-Yuste, V.; Sabate, M.; Sans-Roselló, J.; Sionis, A.; Badimon, L. Circulating microparticle signature in coronary and peripheral blood of ST elevation myocardial infarction patients in relation to pain-to-PCI elapsed time. Int. J. Cardiol. 2016, 202, 378-387. [CrossRef] [PubMed]

86. Santilli, F.; Marchisio, M.; Lanuti, P.; Boccatonda, A.; Miscia, S.; Davì, G. Microparticles as new markers of cardiovascular risk in diabetes and beyond. Thromb. Haemost. 2016, 116, 220-234. [CrossRef] [PubMed]

87. Campello, E.; Radum, C.M.; Duner, E.; Lombardi, A.M.; Spiezia, L.; Bendo, R.; Ferrari, S.; Simioni, P.; Fabris, F. Activated plateletderived and leukocyte-derived circulating microparticles and the risk of thrombosis in heparin-induced thrombocytopenia: A role for PF4-bearing microparticles? Cytom. Part B Clin. Cytom. 2018, 94, 334-341. [CrossRef] [PubMed]

88. Hoeft, F.; Barnea-Goraly, N.; Haas, B.W.; Golarai, G.; Ng, D.; Mills, D.; Korenberg, J.; Bellugi, U.; Galaburda, A.; Reiss, A.L. More Is Not Always Better: Increased Fractional Anisotropy of Superior Longitudinal Fasciculus Associated with Poor Visuospatial Abilities in Williams Syndrome. J. Neurosci. 2007, 27, 11960-11965. [CrossRef] [PubMed]

89. Lawrenz, M.; Brassen, S.; Finsterbusch, J. Microscopic diffusion anisotropy in the human brain: Reproducibility, normal values, and comparison with the fractional anisotropy. Neuroimage 2015, 109, 283-297. [CrossRef] [PubMed]

90. Thomalla, G.; Glauche, V.; Koch, M.A.; Beaulieu, C.; Weiller, C.; Röther, J. Diffusion tensor imaging detects early Wallerian degeneration of the pyramidal tract after ischemic stroke. Neuroimage 2004, 22, 1767-1774. [CrossRef]

91. Song, S.K.; Sun, S.W.; Ju, W.K.; Lin, S.J.; Cross, A.H.; Neufeld, A.H. Diffusion tensor imaging detects and differentiates axon and myelin degeneration in mouse optic nerve after retinal ischemia. Neuroimage 2003, 20, 1714-1722. [CrossRef]

92. Davis, S.W.; Dennis, N.A.; Buchler, N.G.; White, L.E.; Madden, D.J.; Cabeza, R. Assessing the effects of age on long white matter tracts using diffusion tensor tractography. Neuroimage 2009, 46, 530-541. [CrossRef] 